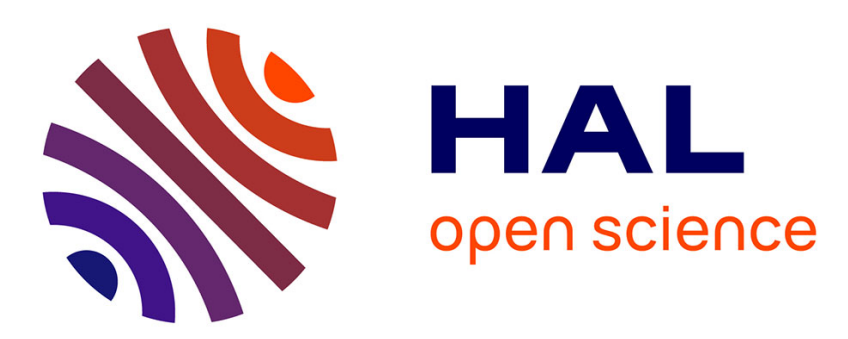

\title{
Quantum Transport on Disordered and Noisy Networks: An Interplay of Structural Complexity and Uncertainty
}

\author{
Mattia Walschaers, Frank Schlawin, Thomas Wellens, Andreas Buchleitner
}

\section{To cite this version:}

Mattia Walschaers, Frank Schlawin, Thomas Wellens, Andreas Buchleitner. Quantum Transport on Disordered and Noisy Networks: An Interplay of Structural Complexity and Uncertainty. Annual Review of Condensed Matter Physics, 2015, 7, pp.223 - 248. 10.1146/annurev-conmatphys-031115011327 . hal-03037584

\section{HAL Id: hal-03037584 https://hal.science/hal-03037584}

Submitted on 2 Apr 2021

HAL is a multi-disciplinary open access archive for the deposit and dissemination of scientific research documents, whether they are published or not. The documents may come from teaching and research institutions in France or abroad, or from public or private research centers.
L'archive ouverte pluridisciplinaire HAL, est destinée au dépôt et à la diffusion de documents scientifiques de niveau recherche, publiés ou non, émanant des établissements d'enseignement et de recherche français ou étrangers, des laboratoires publics ou privés. 


\title{
Quantum Transport on Disordered and Noisy Networks \\ - an Interplay of Structural Complexity and Uncertainty
}

\author{
Mattia Walschaers ${ }^{1,2}$, Frank Schlawin ${ }^{1}$, Thomas \\ Wellens $^{1}$, and Andreas Buchleitner ${ }^{1,3}$ \\ ${ }^{1}$ Institute of Physics, Albert-Ludwigs-Universität, Freiburg i.Br., Federal \\ Republic of Germany, D-79104 \\ ${ }^{2}$ Instituut voor Theoretische Fysica, University of Leuven, Heverlee, Belgium, \\ B-3001 \\ ${ }^{\mathbf{3}}$ Freiburg Institute of Advanced Studies, Albert-Ludwigs-Universität, Freiburg \\ i.Br., Federal Republic of Germany, D-79104
}

Xxxx. Xxx. Xxx. Xxx. YYYY. AA:1-29

This article's doi:

$10.1146 /(($ please add article doi $))$

Copyright @ YYYY by Annual Reviews. All rights reserved

First page note to print below DOI/copyright line.

\section{Keywords}

quantum transport, quantum control through disorder, many-particle transport, indistinguishability, complex materials

\section{Abstract}

We discuss recent research on quantum transport in complex materials, from photosynthetic light harvesting complexes to photonic circuits. We identify finite, disordered networks as the underlying backbone and as a versatile framework to gain insight into the specific potential of nontrivial quantum dynamical effects to characterize and control transport on complex structures. We discriminate authentic quantum properties from classical aspects of complexity, and briefly address the impact of interactions, nonlinearities, and noise. We stress the relevance of what we call the non-asymptotic realm, physical situations where neither the relevant time and length scales, the number of degrees of freedom or constituents tend to very small or very large values, nor do global symmetries or disorder fully govern the dynamics. While largely uncharted territory, we argue that novel, intriguing and non-trivial questions for experimental and theoretical work emerge, with the prospect of a unified understanding of complex quantum transport phenomena in diverse physical settings. 


\section{Contents}

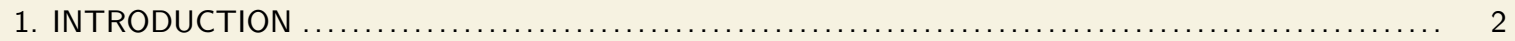

2. EXCITATION TRANSPORT ON NETWORKS WITH CONSTRAINED DISORDER $\ldots \ldots \ldots \ldots \ldots \ldots . . \ldots$

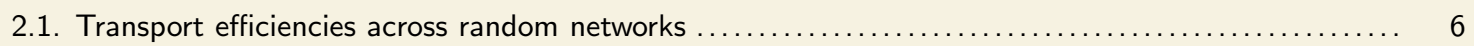

2.2. Constraints to optimize transport across disordered networks $\ldots \ldots \ldots \ldots \ldots \ldots \ldots \ldots \ldots \ldots \ldots \ldots \ldots \ldots$

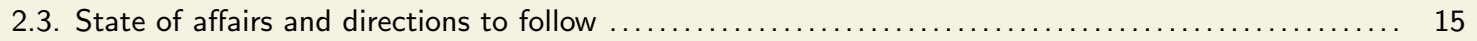

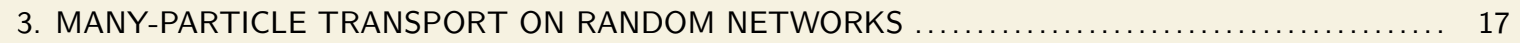

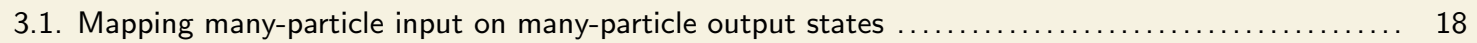

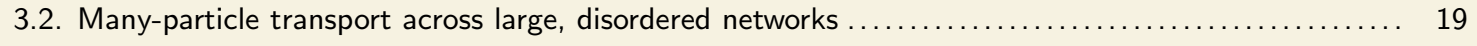

\section{INTRODUCTION}

"Quantum transport" expresses a specific view on the world of quantum phenomena, which originates in condensed matter physics, and, to some extent, physical chemistry: An excitation, an electron or a quasiparticle is to be shipped from input to output of a physical sample to be characterized, possibly by macroscopic quantifiers like its resistivity (1) - or to be transferred from some donor to some acceptor molecular unit, typically over mesoscopic scales, to trigger chemical reactions $(2,3)$. As immediately apparent when defining input and output state asymptotically, this view is intimately related to scattering theory (with the transmission of a plane wave through a potential barrier as the elementary text book example). In an alternative perspective, by its very construction, it defines one of the standard scenarios for non-equilibrium quantum physics. While historically (and anthropomorphically) set in the spatial degree of freedom, transport can, of course, likewise occur in arbitrary directions of phase space, as well as on the energy axis. Often, however, a change of stage implies a change of research community and jargon: Quantum transport on the energy axis (often the momentum axis in disguise) is witnessed and controlled, e.g., in the physics of light matter interaction (from strong to feeble electromagnetic fields), and often comes under the alternative label "quantum dynamics" $(4,5)$. When decomposable into finite numbers of two-level systems or elementary excitations, at a manageable level of complication, "quantum simulation" as a specific brand of "quantum information" sets out to mimic elementary quantum transport phenomena $(6,7)$.

The elementary ingredient of quantum transport is incarnated by Young's double slit, when taken to the granular level of the self-interference of a single particle: While, since Feynman (8), this phenomenon is nowadays very well understood, from the underlying dynamics to the emergence of the interference pattern upon integration over single particle events (9), it gives rise to a panoply of rather dramatic and not-yet-common-sense quantum interference phenomena once the number of interfering amplitudes (and, possibly, particles) is increased. In particular, when we enter the realm of multiple scattering $^{1}$ phenomena (again: possibly, but not necessarily in configuration space), quantum interference effects can prevail even upon averaging over a broad range of, randomly chosen, physical realizations of the scattering potential, ${ }^{2}$ what, on a first glance, ap-

\footnotetext{
${ }^{1}$ Of a single particle on a complicated potential landscape, and/or of many particles.

${ }^{2}$ Defined, in an actual experiment, e.g., by different, macroscopically identically prepared samples; slow drifts of the microscopic sample structure or of externally applied fields; variations of the 
pears very counterintuitive when contemplating the fate of Young's double slit interference pattern upon independent averaging over the slits' positions. Yet, the most prominent such disorder-induced interference effects, weak (10), strong or Anderson (11) and, in a Hamiltonian setting, dynamical (12) localization, bear very tangible macroscopic manifestations, from enhanced back-scattering signals (first observed on Saturn's rings (13)) to by now well-defined and well-controlled metal-insulator quantum phase transitions $(14,15,16,17,18,19,20,21,12,22,23,24,25,26,27,28,29,30,31,32,33,10,34,35)$.

Most importantly, the truly amazing improvement of experimental diagnostic tools and of the experimental control on composite, hybrid quantum systems nowadays allows, arguably for the first time, to systematically explore the controlled transition from manyto single-particle quantum transport and dynamical phenomena $(36,37,38,39$, 40), on potential landscapes which interpolate, possibly on a hierarchy of scales, between perfect symmetries and disorder $(41,42,43,44,45,46)$ Beyond the experimental mimicry of wellestablished theoretical models for extended solids or fields, the truly innovative potential of these rather recent experimental progresses lies in charting the largely unknown territory between single particle quantum dynamics and the thermodynamic limit $(47,48,49,50,39)$, which can be safely predicted to offer plenty of original research questions and surprising answers. Apart from offering novel ways to control (quantum) transport properties which ultimately define the efficiency of technological devices (51) (e.g., for light energy conversion, such as LED or PV), such endeavour will almost certainly qualitatively improve our understanding of the quantum-to-classical transition $(52,53,38)$, as one of the very fundamental open issues of quantum theory (and of its interpretation) to date.

A very useful and versatile scenario for the study of quantum dynamics in what we will here call the non-asymptotic range are finite networks or graphs of variable topology, which can mediate transport of one or more particles $(54,55,56,57,58,59,60,61)$. On the one hand, the network's topology allows a flexible transition from order to disorder, and, on the other, it offers a broad choice of observables to characterize single- or many-particle transport. The network can be connected to leads (supporting continua of scattering states), such as to define a bona fide scattering problem $(57,62,63,64)$, and/or locally or globally interfaced with incoherent environments, such as to incorporate dynamical rather than static disorder - vulgo noise (which, on sufficiently long time scales, kills quantum interference) (65).

The network's topology (beyond, simply, its dimension) can be employed to control the probability of particle encounter, and thus, through the particles' indistinguishability and/or their interaction strength, the structure of the quantum interference terms which control its transport properties $(43,66)$. Furthermore, a network can be considered the natural backbone of a multitude of physical transport problems, from regular or disordered lattices in one, two or three dimensions, in configuration, phase, or energy space $(54,67,68,69,70)$, to molecular aggregates in functional materials or biological substrates $(58,71,72,73)$, quantum algorithms (74), photonic circuits $(75,76)$, electromagnetic transmission lines, multiple scattering media - e.g. cold atomic clouds and Rydberg gases $(50,77,78)$ with slowly (with respect to a single, multiple scattering event) drifting conformations, and many more. The present contribution will exploit this versatility, to illustrate the rich phenomenology of quantum interference effects in the non-asymptotic range, between symmetry and disorder, coherence and noise, distinguishability and discernability. We will

input state or channels; etc.
LED: Light Emitting Diodes

PV: PhotoVoltaics 


\section{STATIC VS. DYNAMICAL NOISE}

There is an important distinction between disorder and noise: Disorder expresses our lack of knowledge of the precise realization e.g. of a potential landscape. Disordered systems evolve unitarily, but to obtain descriptions which are robust against variations of the microscopic hardwiring of the generating Hamiltonian, a disorder average has to be performed. This in general leads to dephasing (or inhomogeneous broadening, in spectroscopy jargon), which, however, can in principle be compensated for by spin echo techniques. In contrast, noise stems from uncontrolled temporal fluctuations of the generating Hamiltonian, on time scales which are shorter than the time scales of interest, and need to be averaged over. This leads to an irreversible loss of information which cannot be recovered, and, in particular, induces decoherence.

ATP: Adenosine Tri Phosphate

LHII: Photosynthetic antenna protein

FMO: Fenna-

Matthews-Olson light harvesting complex

PC645: Phycocyanin 645 import concrete examples from recent scientific debates on the role of quantum coherence in biophysical contexts, and on controlled many-particle interference in photonic circuits, but will also touch upon de facto closely related, while apparently distinct quantum transport phenomena in other (sub-) fields.

\section{EXCITATION TRANSPORT ON NETWORKS WITH CONSTRAINED DISORDER}

We start out with a paradigmatic single particle transport problem on finite networks, which has received renewed interest in the interpretation of spectroscopic data, of unprecedented quality, on photosynthetic functional units of plants, algae, and photosynthetic bacteria $(79,80)$, as well as on photovoltaic blends useful, e.g., for organic photovoltaic devices $(81,82)$. In the photosynthetic scenario, the energy carried by an incoming photon is transformed into an electronic excitation, forming an exciton $(83,84)$, which then needs to be transported to what is known as the reaction centre, a molecular structure where the organism uses that incoming energy to create free charges which then feed the reservoir of ATP as the chemical energy currency. The excitation transport from the absorbing molecular site to the reaction centre is mediated by supramolecular structures which come in very different architectures and with variable (but always clearly finite, in the sense of being far from the thermodynamic limit) size in different species, with a coexistence of symmetric and disordered structures on different (energy, length and time) scales, e.g. in the LHII photosynthetic antenna protein of purple bacteria, or rather as completely disordered 3D structures without apparent symmetries, e.g. in the FMO complex of green sulfur bacteria, or in the cryptophyte PC645 complex of marine algae (85). In general, these supramolecular structures are relatively stiff and exhibit drifts only on time scales much longer than the typical times required for the excitation transport to the reaction centre. However, since these are transport phenomena in living organisms, they occur at finite temperature and are certainly subject to some sort of noise, as well as, possibly, to quasi-deterministic (and hitherto very poorly understood) re-conformations related to their functional role on larger (time and length) scales (86).

By now quite firmly consolidated experimental evidence shows that, notwithstanding (or, probably, because of) their intricate architectures and their embedding in a clearly very noisy macro-structure, these supramolecular transport units indeed can sustain co- 


\section{QUANTUM THEORY AND PHOTOSYNTHESIS}

Ultrafast nonlinear spectroscopy (87) on molecular aggregates such as the FMO complex has revealed signatures of coherent coupling between excitonic states, on transient time scales and at ambient temperatures. This triggered an intense debate on the potential role of nontrivial quantum effects (i.e., quantum phenomena which go beyond defining effective rates which can then be absorbed into effective evolution equations) for the respective biological function. Current candidate scenarios are photosynthesis (79), avian navigation in the Earth's magnetic field (88) and olfaction (89), but conclusive evidence for any functional relevance is missing to date.

herent superpositions of vibrationally dressed excitonic eigenstates, (90) ${ }^{3}$ even at ambient temperatures, on transient time scales, under laboratory conditions (90, 91, 92, 93, 81). What remains, however, a completely open question is whether such quantum superpositions, and the generically associated quantum interference effects, actually do occur in vivo and whether they may be used (whether by nature or by - quantum - engineers) to foster the supramolecular structure's functional purpose.

That this undeniably highly intriguing question did hitherto not receive an answer has essentially two fundamental reasons: On the one hand, given the intrinsic complexity of the object under study, experimentalists have so far been unable to conceive tools of deliberate intervention to enhance or suppress coherences in the supramolecular dynamics, and to unambiguously probe the impact thereof on macroscopic indicators of the transport efficiency, such as, e.g., the quantum efficiency, i.e., the number of charges generated in the reaction centre, per incoming photon. Note that such intervention to control radiation transport in complex materials is at least in principle in reach, as has been experimentally demonstrated with photonic nano-structures (94). On the other hand, theory is so far unable to come up with quantitative predictions which are amenable to direct verification by state of the art experiments, and distinctive enough to discriminate different transport scenarios. Some approaches, based on very advanced computational physics (or chemistry) with considerable computational overhead, consist in consistently reproducing experimental data $(2,3,73)$, such as to extract effective rates etc, though do not offer quantitative predictions which could be falsified by experiment, nor do they help to gain a better understanding of the decisive structural elements which guarantee the functional properties of the molecular machines of interest. Another philosophy attempts to grasp those essential structural or dynamical features, often with simple, strongly reductionist quantum dynamical models $(71,72,69,95,96)$. While this in principle allows to define specific and, possibly, mutually exclusive transport scenarios, these models often lack sufficient detail to match realistic experimental conditions, such that experimental data do not allow to unambiguously discriminate between them. Hence, what is needed are more refined tools for targeted experimental intervention, and more faithful, yet minimalistic theoretical models which al-

\footnotetext{
${ }^{3}$ The strength of the coupling between excitonic and vibrational degrees of freedom is, again, widely variable from species to species. The debate on the prevalence of excitonic or vibrational coherence has however converged by now to some sort of consensus that neither one can be completely neglected, and that one thus often witnesses vibronic coherences.
} 
low to integrate the diverse time, length and energy scales which need to be orchestrated to achieve and quantify certain functional properties.

Nonetheless, the diverse theoretical approaches currently on the market already account for a good part of the basic ingredients which determine the transport efficiency across the light harvesting unit:

- coherent transport on short, transient time scales $(58,60,69,96,95,97)$;

- dominantly incoherent transport on asymptotic, long time scales (the traditional view in chemical physics) (98);

- non-negligible coupling between excitonic and vibrational degrees of freedom (90), or, to some extent equivalently;

- structured spectral densities of the environmental degrees of freedom coupled to the excitonic manifold $(99,100)$;

- statistical variations of transport efficiencies upon sampling over different conformational realizations $(58,97,96,101)$.

Let us now describe an elementary network model which can accommodate these diverse features, inspired by the phenomenology of the FMO unit.

\subsection{Transport efficiencies across random networks}

According to currently available structure data, the FMO is a three dimensional molecular network composed of seven or eight molecular sites defined by the locations of its constituent chlorophyll units (85). In the desire to faithfully represent the FMO's excitonic spectrum (which mediates the excitation transport), the chlorophylls are modelled as dipolar molecules with two electronic (ground and excited) states, which are coupled to each other through dipole-dipole interactions. The relative positions and orientations of the constituent molecular dipoles, the on-site electronic energy levels and the inter-site coupling strengths are tabulated in the literature $(102,103)$. Slight variations between different references and the always relatively large error bars which garnish the tabulated numerical values are an expression of the fact that these are, and, given the complexity of the object of interest, ought to be effective descriptions, which imply strong coarse graining, i.e. an effective average over the many unresolved degrees of freedom of the supramolecular structure. Indeed, these available effective descriptions are deduced by careful deduction of coupling strengths etc from experimental data as well as from state of the art structure calculations. In particular in an interdisciplinary context and discourse it is crucial to fully appreciate this rather fundamental distinction between the experimental and theoretical analysis of quantum dynamics in a physical chemistry or biophysics setting, in contrast to the, by their very construction, highly controlled and engineered objects of quantum optical experiments and theory. ${ }^{4}$ Furthermore, note that, while the chlorophyll molecules which define the network's sites are a priori identical, the onsite energies of the tabulated effective FMO Hamiltonians are not identical, i.e., exhibit some non-trivial potential landscape, which is due to the individual chlorophylls' "local environments" (102, 103). In biophysics jargon, this refers to shifts of the electronic eigenenergies through local couplings to background degrees of freedom which, e.g., span the supramolecular conformation space.

\footnotetext{
${ }^{4}$ Where Hamiltonians are engineered as naked as they stand, with excellent control on the errors incurred by neglect of weakly coupled, well-defined degrees of freedom (104). 
Hence, quantum transport of a single excitation across the FMO molecular network is generated by an effective $N$-site Hamiltonian operating on an $N$-dimensional Hilbert space. At present, it seems to be consensus that it is extremely unlikely that more than one excitation at a time is injected into the network, what also eases dynamical simulations very considerably (also see Sec. 3 below). ${ }^{5}$

Given the above, a natural and popular approach to modelling excitation transport on the FMO network is by quantum dynamical simulation, which essentially propagates a suitably chosen initial condition over relevant time scales (107). In general, this is done by numerical solution of some effective evolution equation for the open system quantum dynamics of the FMO Hamiltonian coupled to some environment with possibly structured spectral density $(71,72,73)$. The structured part of the environment's spectral density accounts for the possibly strong coupling to selected environmental degrees of freedom, which may give rise to non-Markovian memory effects (108) in the open system time evolution (99). An alternative to the description by a non-Markovian environment consists in incorporating the prominent environmental degrees of freedom (which cause the structured component of the spectral density) into the system Hamiltonian (then no more a strictly excitonic Hamiltonian representing one single effective degree of freedom, but "dressed" by a background, e.g., vibrational coordinate, in close analogy to dressed state descriptions of light-matter interaction, e.g. in quantum optics (104) and strong field physics (109)), which then interacts with a Markovian (structure-less) bath (65). Finally, the environment coupling may also incorporate the irreversible dissipation into a sink attached to a specific network site, to account for the desired delivery of the excitation to the reaction centre $(110,111)$.

Such quantum dynamical approach is indeed capable to qualitatively reproduce most experimentally observed features - long-lived coherences between excitonic states, which manifest, e.g., in a damped beating signal of suitably defined correlation functions, nonMarkovian effects induced by a structured (vibrational) environment, noise-induced transport across a disordered network with excitonic eigenstates which are localized on a finite subset of the molecular sites, and coherent transport mediated by vibrational dressing of the excitonic manifold. If excitation transport units like the FMO compound have been optimized by evolution, e.g. for rapid and complete excitation transfer, this picture suggests a carefully tuned interplay of coherent, incoherent and dissipative couplings, to delocalize excitonic transport, bridge energy gaps, and induce directionality towards the reaction centre. However, this is a conclusion based on the simulated dynamics generated by an averaged, effective Hamiltonian, while individual complexes differ in their microscopic structures, giving rise to measurably different (91) dynamical evolutions. One needs to remember then that, in general, the quantum dynamics on disordered systems is not self-averaging, i.e.

$$
\left\langle e^{-i t H}\right\rangle_{\text {disorder }} \neq e^{-i t\langle H\rangle_{\text {disorder }}},
$$

such that the above optimization argument ultimately hinges on the ambient noise overriding the Hamiltonian structure, to induce efficient diffusive, classical transport (112). This is in line with the traditional view that, at ambient temperatures, all transport must ultimately be driven by stochastic activation. Whether classical diffusive transport is com-

\footnotetext{
${ }^{5}$ This observation is also relevant for the discussion whether coherence effects can play a role in vivo, when the sample is excited by sun light, i.e. an incoherent source, rather than by coherent laser radiation $(105,106)$.
} 


\section{WEAK AND STRONG LOCALIZATION}

Weak and strong localization are well-defined concepts in condensed matter and mesoscopic transport theory $(1,42)$. They describe the impact of disorder-induced quantum interference effects on the transmission probability across disordered potential landscapes, which survive the disorder average and can be rather dramatic. Often it is difficult to distinguish interference-induced (weak or strong) localization proper from other mechanisms which impede transport, such as, e.g., (partial) phase space barriers or the somewhat trivial confinement of an eigenstate to one or few local potential minima. Consequently, the terminology tends to be used in a rather fuzzy manner in the literature beyond condensed matter and mesoscopics.

\section{Weak localization phenomena}

Weak localization consists in a reduction of the diffusion constant, hence of the transmission probability, due to an enhanced return probability to a given point within the sample. The latter is a consequence of the interference of time reversed multiple scattering paths, and also leads to (enhanced) coherent back scattering of the injected probability flux. Weak localization is often considered to be a precursor of strong localization, in the regime of weak disorder, i.e. for $\ell k \gg 1$, with $\ell$ the scattering mean free path and $k$ the transported particle's wave vector.

\section{Strong localization phenomena}

Strong or Anderson localization consists in the vanishing of the diffusion constant, as a consequence of complete, destructive interference upon transmission. It manifests differently in one, two and three dimensions and is associated with a quantum phase transition from delocalized to exponentially localized eigenstates on $3 \mathrm{D}$ disorder potentials, at $\ell k \simeq 1$. An analogous phenomenon in light-matter interaction at high spectral densities, dubbed "dynamical localization", leads to a suppression of the effective energy transfer from the driving to the matter degree of freedom.

\section{Control of localization phenomena}

Due to the dramatic impact of localization phenomena on dynamical and spectral features, efforts are recently made to exploit or manipulate these effects for the purpose of quantum control, e.g. by tailoring inter-site couplings or by injecting nonlinearities $(45,94)$.

patible with experimentally observed time scales remains a matter of controversial debate in the literature $(113,114,115)$.

Another approach to revealing the potential of non-trivial quantum effects to enhance the functionality of molecular networks à la FMO is statistical rather than dynamical in spirit. In terms of the above, the object of interest is the statistical distribution of transport coefficients as generated by $e^{-i t H}$, when sampling over the different physical realizations of $H$ as present in the given organism. The underlying intuition stems from the quantum transport theory of finite, disordered systems: Since disorder-induced quantum interference effects as Anderson localization are a consequence of destructive multi-path interference upon transmission, characteristic transport coefficients will exhibit strong fluctuations from realization to realization (since each realization defines a different interference condition), for a finite sample length (116). Indeed, in the Anderson case these fluctua-

8 Mattia Walschaers, Frank Schlawin, Thomas Wellens, and Andreas Buchleitner 
tions are exponentially large, and define an unambiguous hallmark of quantum interference (117). Consequently, in disordered, finite networks there must be finite probability for strongly interference-enhanced transmission probabilities. At least on transient time scales (on which, in a noisy environment, quantum coherence can at best survive), this will be qualitatively unaffected by the ambient noise, which, however, will overtake on asymptotic time scales (111).

While dipole-coupled networks as realized by the molecular aggregates we are here interested in do not give rise to Anderson localization in a strict sense (since this type of inter-site coupling does not induce exponentially localized eigenstates (78)), they still give rise to multipath interference, with relative phases which must sensibly depend on the specific conformation of the network. If we model the network Hamiltonian as

$$
H=\sum_{i \neq j=1}^{N} v_{i, j}|j\rangle\langle i|,
$$

where the sum runs over all pairs of distinct $N$ network sites ${ }^{6}$ and the inter-site dipoledipole coupling $v_{i, j}$ is determined by distance and relative dipole orientation, different conformations will lead to different realizations of the Hamiltonian. Such Hamiltonians (2) generate excitonic dynamics on a fully connected random network, where an excitation is destroyed at site $i$ and created at site $j$, mediated by the coupling strength $v_{i, j}$. Energy is transferred across the network alike a quasi-particle.

In contrast to the above quantum dynamical point of view, which considers the average Hamiltonian structure as the actual structure generating the dynamics, one can now adopt the somewhat complementary point of view and assume that the complex' conformations are randomly distributed, e.g. within a sphere where input (where the photon is absorbed) and output (where the excitation is eventually delivered to the reaction centre) site are fixed at north and south pole, respectively. Thus, random positioning of the intermediate chlorophyll sites induces a random distribution of the coupling matrix elements in (2), through $v_{i, j} \propto r_{i, j}^{-3}$ (if the relative dipole orientations are neglected, which does not change the qualitative picture (58)), and thus gives rise to conformation dependent transport coefficients. If the latter are defined by what has been baptized the transfer efficiency (69)

$$
\left.\mathcal{P}_{H}=\max _{t \in\left[0, T_{R}\right)} \mid\left.\left\langle\text { out }\left|e^{-i H t}\right| \phi(0)\right\rangle\right|^{2},|\phi(0)\rangle=\mid \text { in }\right\rangle
$$

with $T_{R}$ a suitably defined benchmark time (see below), $\mathcal{P}_{H}$ must exhibit strong fluctuations under random changes of the network conformation, according to the above intuition, and as indeed confirmed by numerical simulations as displayed in Fig. 1.

What becomes evident from these results is that, while the average transfer efficiency over the entire statistical sample of Hamiltonians (2) is indeed low (approx. 5\%), as expected from common sense expectations on the localization properties of excitonic eigenstates on random structures, there does exist a non-negligible sub-set of Hamiltonian realizations which give rise to transfer efficiencies much above average, and in some cases close to unity (somewhere between 50 and 100\%). Since, under conditions of strictly unitary transport as here assumed, the transfer efficiency is essentially given by the spectral decomposition of

\footnotetext{
${ }^{6}$ Including the input and output sites, $\mid$ in $\rangle=|1\rangle$ and $\mid$ out $\rangle=|N\rangle$.
} 


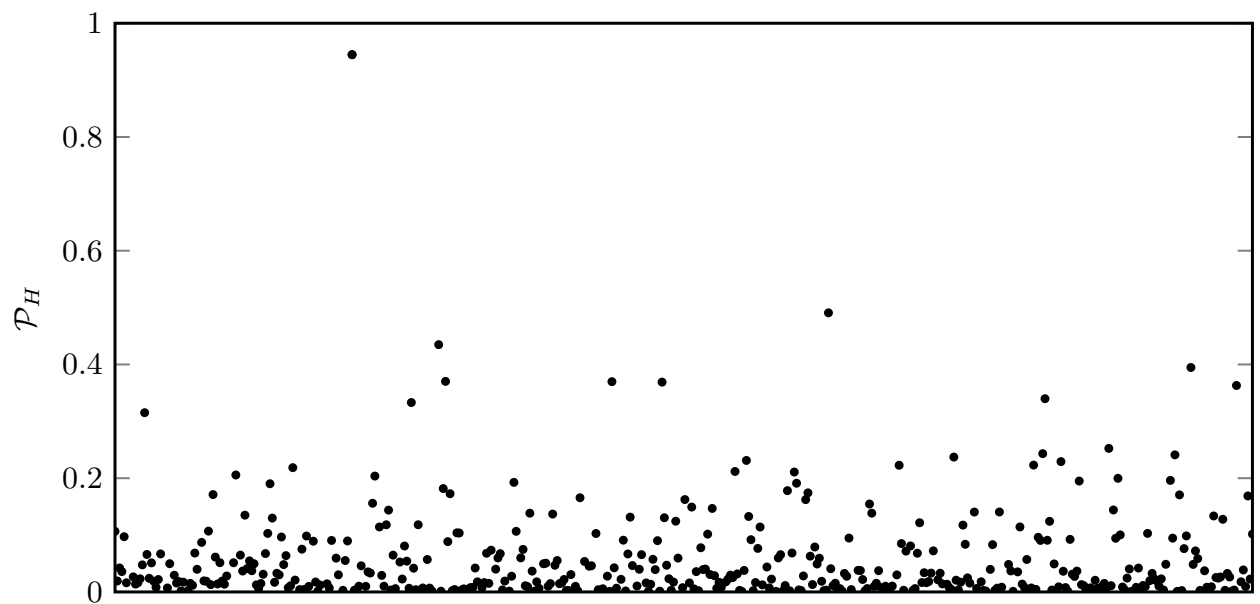

random realization

Figure 1

Excitation transfer efficiencies $\mathcal{P}_{H}$, as defined by (3), across random realizations of an $N=7$ sites random network (69). The latter is described by Eq. (2), with $T_{R}=\pi / 20|V|, V=v_{\text {in,out }}$, input and output site located at the north and south pole of a sphere, and the intermediate sites, $N=2, \ldots, 6$, randomly distributed within the sphere, to randomize the coupling matrix elements $v_{i, j}$ in Eq. (2). While the majority of network realizations gives rise to rather moderate transfer efficiencies $\mathcal{P}_{H} \simeq 5 \%$, there is a finite sub-set of realizations which generate significantly more efficient transport, up to values not too far from unity. Since the dynamics is purely coherent, this is a result of constructive multi-path interference between the different transmission amplitudes connecting input and output site.

the unitary generated by $H$,

$$
\left.\mid\left.\langle\text { out }|U(t)| \text { in }\rangle\right|^{2}=\mid \sum_{j}\left\langle\text { out } \mid \eta_{j}\right\rangle\left\langle\eta_{j}\right| \text { in }\right\rangle\left.\exp \left(-i E_{j} t / \hbar\right)\right|^{2}
$$

such high transfer efficiencies imply the existence of at least one excitonic eigenstate $\left|\eta_{j}\right\rangle$ which exhibits an appreciable overlap with, both, |in $\rangle$ and $\mid$ out $\rangle$.

\subsection{Constraints to optimize transport across disordered networks}

It now immediately comes to mind to optimize a given random Hamiltonian with respect to the transfer efficiency, and, indeed, genetic algorithms (118) seeded with any one of the Hamiltonians which give rise to typical transfer efficiencies in Fig. 1 rapidly converge into strictly optimal Hamiltonians, which very quickly depopulate the input site, and coherently feed all population into the output site, see Fig. 2 (58). But which are the specific features which render such Hamiltonians optimal? When inspecting their spatial structure, no apparent symmetries stand out, and the problem is in this sense somewhat reminiscent of the optimal pulse shapes generated by genetic algorithms (119) or other optimization strategies (120) for optimal coherent control: Also there, the generated optimal pulse shapes are in general little telling on which underlying principle defines the optimal superposition of paths in state space, to optimize the given target functional. However, the time dependence of the different sites' populations as displayed in Fig. 2 exhibits such sought-for symmetry, 


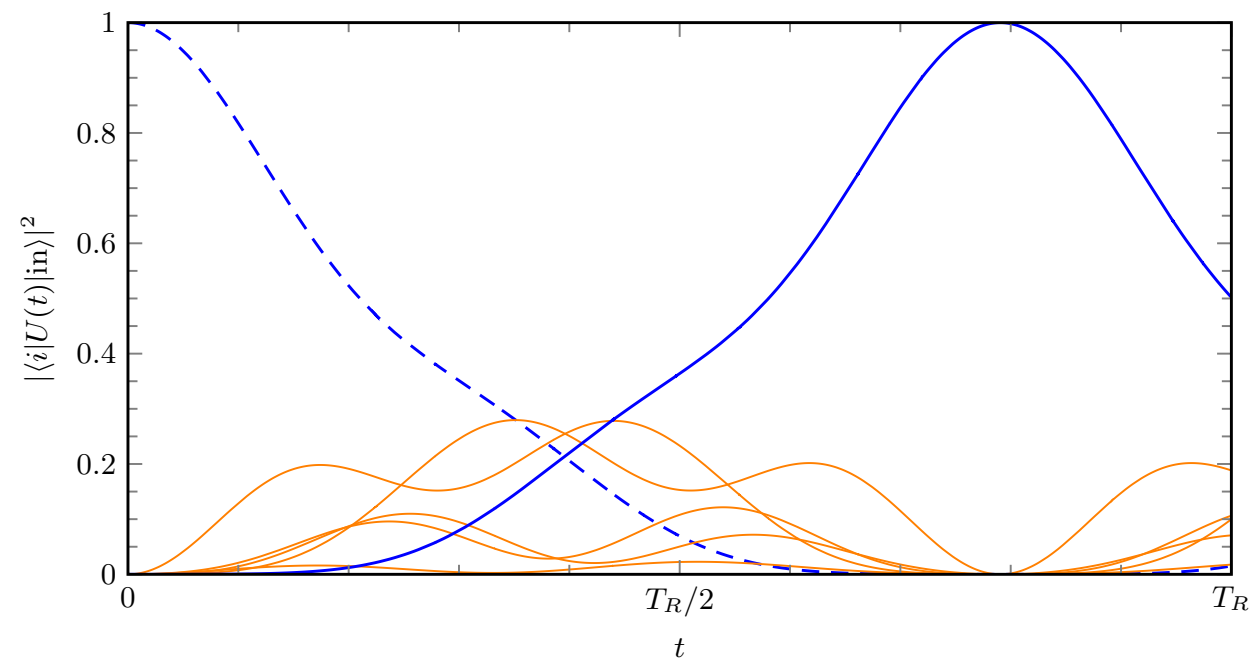

Figure 2

Populations of input (blue dashed), output (blue solid) and bulk (orange) sites, as a function of time (in units of the benchmark time $T_{R}$ ), for a $N=7$ network optimized by a generic algorithm seeded with a typical low-efficiency configuration as represented by low values of $\mathcal{P}_{H}$ in Fig. 1

(58). Since the dynamics is purely coherent, the observed time evolution must be quasi-periodic. Strikingly, the populations are indeed periodic, and (approximately) mirror-symmetric with respect to a time $t^{*} / 2$, i.e. $|\langle i \mid \phi(t)\rangle|^{2} \simeq\left|\left\langle J i \mid \phi\left(t^{*} / 2-t\right)\right\rangle\right|^{2}$. The time evolution of input and output site are strongly reminiscent of the dynamics of a wave packet in a double well potential. The mirror symmetry on the time axis is suggestive of a centrosymmetric structure of the underlying Hamiltonian, while the effective double well dynamics is indicative of a dominant doublet in the spectral structure, with a weight $\alpha$, see Eq. (6), which is determined by the relative amplitudes of the input and output site populations as compared to that of the bulk states.

with the additional prominent feature that the input and output site's populations largely dominate over those of the intermediate sites (which, nonetheless, and importantly, remain non-negligible). A sufficient condition to generate such symmetry on the time axis is the centrosymmetry (121) of the underlying Hamiltonian, i.e. the property that $H$ be symmetric under mirroring with respect to its center, for some labelling of the network sites. More formally speaking, this is tantamount of the commutation relation $J H=H J$, with the exchange operator $J_{i, j}=\delta_{i, N-j+1}$, together with $\mid$ in $\rangle=J \mid$ out $\rangle$. A possible measure of centrosymmetry is then given by

$$
\epsilon=\frac{1}{N} \min _{\sigma}\left\|H-J^{-1} H J\right\|,
$$

where minimization is performed over permutations $\sigma$ of the intermediate sites $2, \ldots, N-1$, ||.|| denotes the Hilbert-Schmidt-norm, and small values of $\epsilon$ correspond to pronounced centrosymmetry, and vice versa. (5) now allows to inspect the correlation between centrosymmetry and transfer efficiency, as done in Fig. 3: Indeed, the correlation is unambiguous, and shows that centrosymmetry of $H$ is a design principle which is very favourable to improve the transport properties of the network. Yet, it is not sufficient, as the distribution of transfer efficiencies for given $\epsilon$ still almost covers the entire interval $[0 ; 1]$.

Here helps the second observation from above: Not only are the populations of the

www.annualreviews.org $\bullet$ Quantum Transport on Disordered and Noisy Networks 


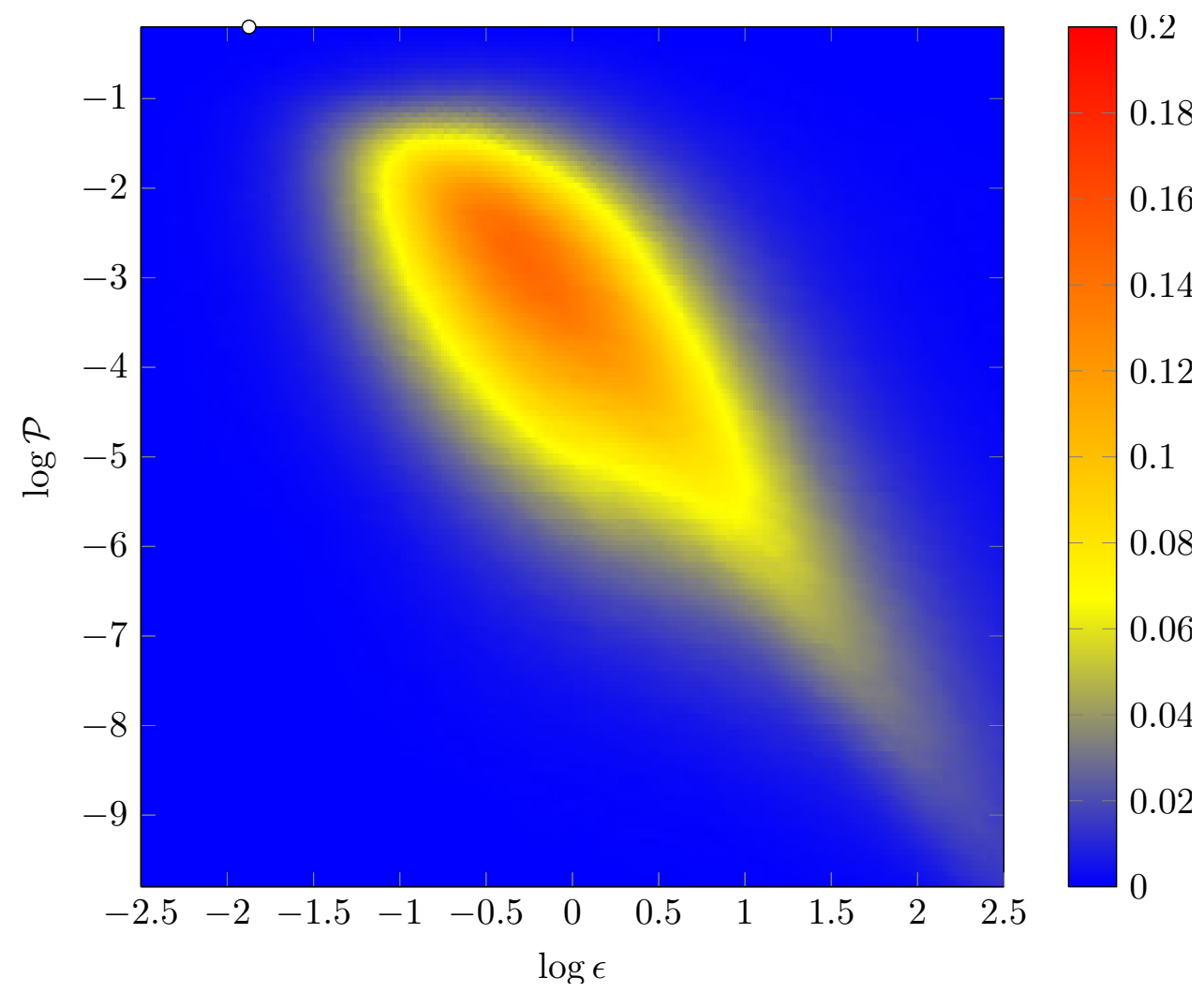

Figure 3

Correlation between the network's centrosymmetry, quantified by $\epsilon$ as defined by (5) - the smaller $\epsilon$, the more centrosymmetric the network - and its transfer efficiency $\mathcal{P}_{H}$ as defined by (3), in a double logarithmic plot. The correlation is unambiguous and confirms that centrosymmetry much favours the probability of efficient excitation transfer. Yet, centrosymmetry does not allow for a safe bet. An additional design principle is needed. The observed correlation is a consequence of quantum interference upon transmission across the network, and fades away under noise (97). The white dot on the top left of the plot represents the optimized network configuration which generates the time evolution displayed in Fig. 2.

network's sites symmetric on the time axis, but also the populations of input and output site dominate over the intermediate sites' populations, at almost all times (except $t \simeq t^{*} / 2$, see Fig. 2). Furthermore, if taken alone, the time evolution of input and output site population is almost a textbook example of the time evolution of the populations of a double well's right and left site when initiated, e.g., on the left. Therefore, what we are facing in the case of optimal transport is an effective double well dynamics - in chemical jargon connecting the donor and the acceptor site - with the intermediate sites simply adjusting the tunneling barrier. Consequently, the eigenvectors $\left|\tilde{\eta}_{j}\right\rangle$ of $H$ are expected to exhibit a dominant doublet $|\tilde{ \pm}\rangle$ with weight

$$
\alpha=|\langle\tilde{ \pm} \mid \pm\rangle|^{2} \gg\left|\left\langle\tilde{\eta_{j}} \mid \pm\right\rangle\right|^{2}, \forall \tilde{\eta}_{j} \neq \tilde{ \pm},
$$

where $| \pm\rangle=(\mid$ in $\rangle \pm \mid$ out $\rangle) / \sqrt{2}$. (6) defines a second design principle, beyond the above centrosymmetry. 
With this intuition gained from the simulation of excitation transport across random networks of type (2), one can now formulate a transport theory for finite size random Hamiltonians with a structure constrained by the above design principles (61). In order to ease a (still rather non-trivial) analytical treatment, we however abandon the geometrical construction of random Hamiltonians via randomized relative positions $r_{i, j}$ within a sphere, and rather employ $N \times N$ Hamiltonians from the Gaussian Orthogonal Ensemble (122) (GOE) of Random Matrix Theory (RMT).

For each realization, $\mid$ in $\rangle$ and $\mid$ out $\rangle$, fulfilling the constraint $\mid$ in $\rangle=J \mid$ out $\rangle$, are associated with those sites $i$ and $N-i+1$ which exhibit the weakest direct coupling matrix element $V=$ $\min _{i}\left|H_{i, N-i+1}\right|$. A thus generated random Hamiltonian constrained by centrosymmetry and the presence of a dominant doublet has the general structure

$$
H=\left(\begin{array}{cccc}
E+V & \left\langle\mathcal{V}^{+}\right| & \\
\left|\mathcal{V}^{+}\right\rangle & H_{\text {sub }}^{+} & \\
& & E-V & \left\langle\mathcal{V}^{-}\right| \\
& & \left|\mathcal{V}^{-}\right\rangle & H_{\text {sub }}^{-}
\end{array}\right)
$$

with $\langle \pm|H| \pm\rangle=E \pm V$, and $\left|\mathcal{V}^{ \pm}\right\rangle$random vectors with Gaussian distributed entries, which mediate the coupling between the dominant doublet and the eigenstates of the GOE submatrices $H_{\text {sub }}^{ \pm}$. The Hamiltonian's specific block-diagonal structure is inherited from its representation in the centrosymmetry eigenbasis: Since $H J=J H$, these operators share a joint set of eigenstates, and the degeneracy of $J$ thus induces the block-diagonal structure of $H$. Phrased differently, the centrosymmetry introduces parity as a quantum number. Note that the upper block of (7), indicated by "+", represents the even parity eigenstates of the network, while the block labeled by "-" depicts the odd parity subspace. The representation in the centrosymmetry (or parity) eigenbasis is crucial for these structures to emerge.

Since the dominant doublet weight $\alpha$ needs to be close to unity, by its very definition, the coupling to the random states associated with the randomly coupled intermediate molecular sites is weak, and can be accounted for perturbatively. Despite being weakly coupled, the collective effect of the intermediate sites amounts to a shift of the doublet states which, if garnished with the proper sign, can enormously enhance the unperturbed, direct tunnel splitting $V$, even in the limit of vanishing direct coupling. Since the tunnelling time is just the inverse of the effective tunnelling splitting, this amounts to a potentially strongly accelerated tunnelling process, which possibly allows to prevail over the time scales of competing loss processes (e.g. recombination of the excitation, not incorporated in the above Hamiltonian), and, thus, to achieve what quantum opticians dub "strong coupling" (104). Note that this scenario is an incidence of what has been conceived as chaos assisted tunneling (CAT) (123) in the quantum chaos literature: If direct tunnelling between the potential wells of a symmetric double well in one degree of freedom is strongly suppressed, the nonlinear coupling to a second degree of freedom may generate "chaotic" eigenstates (124) which sensibly depend on a control parameter and are themselves weakly coupled to the original tunnelling doublet. The resulting, effective tunnelling rate between the wells can then be shown to exhibit very strong fluctuations under changes of the control parameter, and may be enhanced by orders of magnitude, through the weak coupling to the chaotic states. In our present example, the random positioning of the intermediate sites, modelled by GOE matrices, mimics the chaoticity induced by the coupling to the additional degree of freedom in the CAT scenario, which is perfectly adequate, in view of the to date
GOE: Gaussian

Orthogonal

Ensemble

RMT: Random

Matrix Theory
CAT: Chaos Assisted Tunneling 


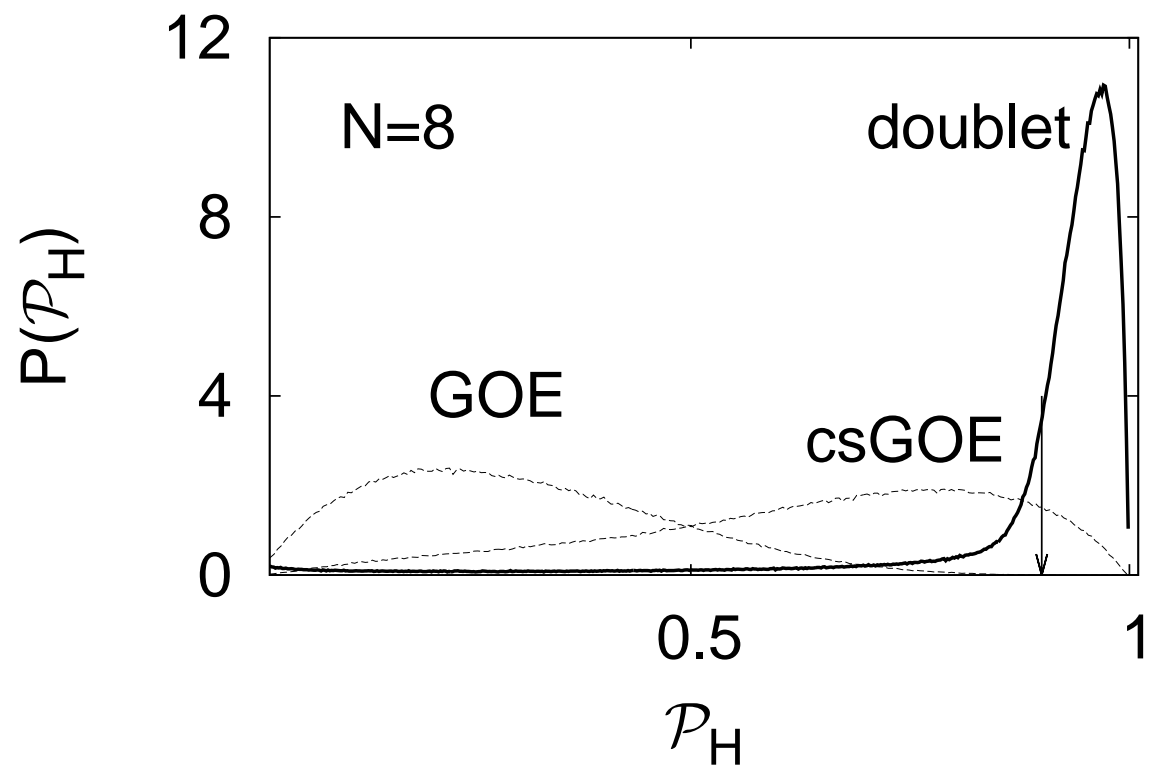

Figure 4

Control of the distribution of transfer efficiencies (with $T_{R}=\pi / 2|V|$ ) of $N=8$ site random networks sampled from GOE matrices, through additional constraints. While the unconstrained GOE ensemble gives rise to a broad distribution of transfer efficiencies with its center in the vicinity of $20 \%$, the centrosymmetry constraint (as quantified by Eq. (5)) generates the csGOE ensemble with a still broad distribution of efficiencies, though with a maximum clearly shifted to appreciable values around $80 \%$. When those centrosymmetric networks are post-selected which on top exhibit a dominant doublet with weight $\alpha=0.9$, see Eq. (6), the distribution narrows down dramatically and allows for almost deterministic delivery of the excitation at the output site, without control of the microscopic positioning of the bulk sites. Only the average density of states in the bulk, the number of network sites, and the average coupling strength between bulk and inand output sites suffice to fix the distribution. An RMT treatment $(96,61)$ allows to predict a lower bound for the maximum of the dominant doublet distribution, here marked by an arrow.

very well verified Bohigas-Giannoni-Schmidt conjecture (125). Indeed, as discussed earlier, statistical scatter of the entries of the effective Hamiltonians of FMO like complexes is due to changes of the local environment as defined by the molecular complexes' conformational structures, which amounts to nothing else but the coupling to other degrees of freedom (e.g., of vibrational character). This statistical scatter is in the present statistical model generated by random sampling over the GOE ensemble.

Given Eq. (7), it is possible to generalize the random matrix theory of CAT for our present purposes, and to derive the distribution of excitation transfer times and efficiencies, as well as the scaling behaviour thereof with the size of the network (either at fixed spectral density or at fixed direct tunnelling coupling $V$ ) (61). Here we only illustrate the effect of the above design principles on the distribution of transfer efficiencies, in Fig. 4. While an unconstrained GOE random network generates a broad distribution of transfer efficiencies

Mattia Walschaers, Frank Schlawin, Thomas Wellens, and Andreas Buchleitner 
with rather unsatisfactory average performance, centrosymmetry shifts this distribution to larger average values, though without much narrowing. The dominant doublet requirement, however, induces a dramatic sharpening of the distribution, which is sharply peaked above $\mathcal{P}_{H}>2 \alpha-1$, thus leading to an almost deterministic transport from input to output site, without precise knowledge of the positioning of the intermediate sites. Only coarse grained quantities fix this dynamical behaviour: the average level density of the bulk states (essentially controlled by the packing of the molecular sites), the average coupling strength of in- and output site to the bulk, and the dominant doublet strength $\alpha$. Note that, while it may appear obvious that a doublet structure can guarantee deterministic transport from donor to acceptor, the mechanism described above allows to embed such structure into a random network of $N$ sites, without appreciable change of the relevant properties of the doublet's eigenvectors, while strongly shortening the transfer times. No detailed knowledge on the microscopic hardwiring of the entire structure is needed, except for the centrosymmetry requirement. In particular, this mechanism allows for a robust implementation of interference-induced enhancement of the transfer efficiency, since only the above-mentioned coarse grained parameters need to be under control.

Let us stress that the above, CAT-inspired transport optimization scheme strictly relies on constructive multipath quantum interference upon transmission across the network, and cannot be reproduced by classically diffusive transport. This can be qualitatively verified by adding, e.g., dephasing noise locally at each molecular site. Once the noise is strong enough to induce approx. one incoherent event per coherent transfer time across the sample, the above correlation between centrosymmetry and transfer efficiency fades away, and the coherent tunnelling between donor and acceptor starts to be corrupted (97). Due to its strictly coherent character, CAT optimization furthermore only induces rapid oscillations of the excitation between donor and acceptor, and delivery to the sink - e.g. through connection to a lead - is not incorporated into the model. However, it can be shown that weak coupling to a sink will not qualitatively alter the dynamics but simply turn the system's excitonic eigenstates into resonances with small decay rates, the latter then feeding the reaction centre through the sink $(58,111)$. This is a well established scenario in mesoscopic physics and light matter interaction, where weak coupling to a continuum allows to probe bound state dynamics without much perturbing it (126). Yet, it is presently not well documented how excitations actually are fed into and extracted from FMO like molecular networks. One may think of slow, directional leakage (occurring over many near-coherent oscillations between donor and acceptor), what is easy to implement since it only requires weak coupling between, e.g., the output site and the reaction centre, as well as, at least in principle, of precisely timed extraction by molecular re-conformation when the excitation arrives at the output site. The latter would require a much higher level of orchestration of excitonic and conformational dynamics than the former, but given the in many respects stunningly high degree of specialization of these biological functional units, it may appear premature to fully exclude such a scenario.

\subsection{State of affairs and directions to follow}

Where do we stand? Beating signals from nonlinear spectroscopy on photosynthetic light harvesting units gave us new, intriguing reasons to think anew about the interplay of disorder, interference and noise when it comes to describing and controlling quantum transport on finite size networks embedded in noisy environments. As far as the transport of single

www.annualreviews.org • Quantum Transport on Disordered and Noisy Networks 
STED: Stimulated emission depletion excitations is concerned, it is now clear that constrained disorder allows rather impressive and robust control of transport efficiencies on short time scales, while noise takes over on long time scales, and overwrites the underlying Hamiltonian structures (by mixing the associated eigenstates). If constraints like the ones suggested above are relevant for the observed transfer efficiencies, then characteristic traits thereof must emerge from spectroscopic data even upon averaging over different realizations of the microscopic network structure, and some recent experimental findings could possibly be interpreted in favour of such hypothesis, inasmuch as certain transition frequencies are robust against disorder averaging in recent spectroscopic data (92). Also statistical spread of coherent transfer time scales has been unambiguously observed in single molecule experiments (91), and thus appears to favour the relevance of the distribution of microscopic Hamiltonian structures rather than noise enhanced transport on the averaged structure. On the other hand, producing large statistical data sets on biological samples is a truly difficult experimental challenge, and a comparison between theory and experiment is therefore uncertain ${ }^{7}$. Furthermore, given the immense variety of light harvesting units implemented by nature, it appears at our present state of knowledge inappropriate to discard any of the proposed mechanisms - be it classical, i.e. noise-, or quantum, i.e. interference-induced - right away.

Still, neither one of these models is so far able to come up with distinctive and experimentally verifiable predictions. On the experimental side, this is due to so far suboptimal control of initial conditions, the lack of tools for selective intervention (such as controlled, locally induced non-linearities (94) or a controlled admixture of noise (128)), and limitations in resolution (note, however, STED (129), even given that its current version requires light intensities which would damage the samples here under study). Somewhat worse, on the theoretical side, it is clear that purely dynamical approaches will be unfit to obtain a qualitative understanding of the working principles of biological functional units, simply due to the underlying complexity and the concommittent statistical variation. Statistical and dynamical tools will need to be merged. Furthermore, models as the ones sketched above need to be enlarged to explicitly incorporate those structures which feed the absorbed photon's energy into the molecular network, as well as the connection to and the charge separation within the reaction center. This could define a first model of sufficient complexity to access, perhaps even quantitatively, one of the decisive, experimentally accessible figures of merit, the quantum efficiency, i.e. the number of charges produced per incoming photon. In addition, such modelling would mimic transport and conversion dynamics on broadly distributed time scales (from fs to ns), with quantum effects certainly confined to the shorter ones. Since much of the excitement about excitation transport in light harvesting machines stems from the possible functional relevance of non-trivial quantum effects, this is likely to be the ultimate challenge, as one needs to establish that the macroscopically observed quantum efficiency sensibly depends on a quantum coherent feature on microscopic length and time scales. At our present state of knowledge, quantum speed-up of excitation transfer on the scale of molecular transport complexes like FMO, as e.g. provided by the above CAT mechanism, would on large (length and time scales) just define a distribution of effective transfer rates, with the distribution itself being truly quantum (since brought about by quantum interference, somewhat similar to mesoscopic conductance fluctuations (130) or Ericson fluctuations (131)). However, it is then to be shown that such specific (quantum) type of distribution hardwires a functional advantage on large scales.

\footnotetext{
${ }^{7}$ But may be in reach (127).
} 
Finally, there are certain more or less tacit assumptions which await clarification. One relevant and actually also fundamentally interesting issue is the difference between photons coming from the sun as compared to those coming from a laser source in the lab $(101,113$, $114,105,132,106)$. When analyzing the steady state of an FMO like structure under solar irradiation it is relatively straightforward to see that coherences cannot be sustained. On the other hand, it is also clear that, if these molecular complexes process excitations one by one, we are not talking about characterizing a non-equilibrium steady state, but rather about coherences on transient time scales. Whether such transient coherences can be induced by the absorption of a single photon remains to be analysed in detail (though all the technical tools are available to do so). Another interesting issue, for the understanding but possibly also for control of the debated transport processes is the role of nonlinearities, due, e.g., to double excitations (133). Can such events, even if rare, affect the transport efficiency, and can deliberately induced double excitations be used as an experimental diagnostic tool? ${ }^{8}$

\section{MANY-PARTICLE TRANSPORT ON RANDOM NETWORKS}

Above, we inspected quantum transport of a single excitation, across a finite, disordered network, and analyzed the impact of additional constraints on the statistics of a characteristic transport quantifier. In closing, we did also remark that one of the open questions to be addressed is the influence of non-linearities on the transport properties. One possibility to create non-linearities is by feeding more than one excitation or particle into the network. Indeed, the quantum dynamics of interacting many particle systems, on regular or disordered lattices, is a long-standing, multi-facetted, and highly non-trivial research area $(136,137,138,139)$. It recently enjoys some renewed interest, not least due to the availability of experimentally controlled many-particle systems of diverse nature $(140,141,142,143,144,145,146)$. Depending on the system's size and on the physical nature of its constituents, different theoretical toolboxes are employed, and blended in the different communities' jargon. However, in substance, the relevant unsolved questions remain:

- How do many-particle interactions or non-linearities affect quantum transport phenomena (49) - on regular as well as on disordered lattices? ${ }^{9}$

- How do statistically robust, macroscopic observables emerge from the microscopic description of many-particle quantum systems of increasing complexity, in equilibrium and out of equilibrium? What is, eventually, the specific role of interactions/nonlinearities $(52,48)$ ?

- What is the potential of controlled nonlinearities as a means to control quantum transport (94), in particular in complex structures?

Rather than following this line of thought, which arguably merits an independent review, we will here take one step back, and acknowledge another feature which automatically comes

\footnotetext{
${ }^{8}$ Compare, e.g., Coulomb and Rydberg blockade effects in mesoscopics (134) and quantum optics (135).

${ }^{9}$ Note that, on the level of the fundamental equations, nonlinearities in interacting cold matter systems have many similarities to well-known phenomena in nonlinear optics, and that nonlinearities impact, actually in a similar, maybe universal manner, on quantum transport in a priori very distinct physical settings, from light matter interaction over condensed, possibly soft matter and mesoscopic physics to quantum simulations!
} 
into play when injecting more than one particle, which is fundamentally quantum in its very nature - rather than the perfectly classical notion of interactions -, and prevails also in the absence of the latter: the indistinguishability of identical particles. Even in the absence of particle-particle interaction does the indistinguishability multiply the alternatives which mediate transport from input to output of some device or network. Consequently, the amplitudes associated with these alternatives need to be summed up coherently to infer the probability of a certain transmission event. In other words, now Young's double slit is no more the only elementary building block, as in the previous chapter, since not only single particle amplitudes will interfere with each other, but also many-particle amplitudes. This generates some rather unexpected effects, even in the absence of interactions, for relatively small particle numbers, and on small, regular networks $(38,43,147,37,148,149)$. Furthermore, the number of interfering alternatives grows very rapidly with the particle number and thus defines an interesting incident of indistinguishability-induced "complexity" (149, 150).

\subsection{Mapping many-particle input on many-particle output states}

Let us thus consider our transport problem under a slightly different perspective now, in second quantized form. The network be described by a unitary matrix $U$ which maps input channels $i$ on output channels $o$, via

$$
a_{i}^{\dagger} \rightarrow \sum_{o} U_{i, o} b_{o}^{\dagger}
$$

where the physical nature of the indistinguishable particles (fermions or bosons) is implied through the (anti-) commutation relations of the creation and annihilation operators $a_{i}^{\dagger}, b_{o}^{\dagger}$ and $a_{i}, b_{o}$ respectively. The symmetries and/or irregularities of the network are determined by the complex-valued entries of $U$. If we prepare an input state |in $\rangle$ by distributing $N$ particles over $M \geq N$ input channels, such that at most one particle is injected into a channel (this allows for a direct comparison of fermionic and bosonic transport), i.e.,

$$
\mid \text { in }\rangle=a_{i_{1}}^{\dagger} \ldots a_{i_{N}}^{\dagger}|\Omega\rangle, i_{j} \neq i_{k} \forall j \neq k
$$

with $|\Omega\rangle$ the $M$-channel vacuum state, then, by virtue of (8), the $M$-channel output state reads

$$
\mid \text { out }\rangle=\mathcal{U} \mid \text { in }\rangle=\sum_{o_{1} \ldots o_{N}=1}^{M} U_{i_{1}, o_{1}} b_{o_{1}}^{\dagger} \ldots U_{i_{N}, o_{N}} b_{o_{N}}^{\dagger}|\Omega\rangle .
$$

This also defines $\mathcal{U}$, the unitary operator on Fock space which implements the map (8).

For the minimal scenario with $M=N=2$ and $U$ representing a balanced beam splitter,

$$
a_{1}^{\dagger} \rightarrow\left(b_{1}^{\dagger}+b_{2}^{\dagger}\right) / \sqrt{2}, a_{2}^{\dagger} \rightarrow\left(b_{1}^{\dagger}-b_{2}^{\dagger}\right) / \sqrt{2},
$$

with one photon (thus, bosonic commutation rules) injected into each of the input modes, this induces the following mapping from input to output state:

$$
|1,1\rangle=a_{1}^{\dagger} a_{2}^{\dagger}|\Omega\rangle \rightarrow \frac{1}{2}\left(\left(b_{1}^{\dagger}\right)^{2}-b_{1}^{\dagger} b_{2}^{\dagger}+b_{2}^{\dagger} b_{1}^{\dagger}-\left(b_{2}^{\dagger}\right)^{2}\right)=\frac{1}{2}\left(\left(b_{1}^{\dagger}\right)^{2}-\left(b_{2}^{\dagger}\right)^{2}\right)=\frac{1}{2}(|2,0\rangle-|0,2\rangle),
$$

with vanishing probability for the coincident output event $\mid$ out $\rangle=|1,1\rangle$, where one photon is detected in each output mode. This is a consequence of the destructive interference

Mattia Walschaers, Frank Schlawin, Thomas Wellens, and Andreas Buchleitner 
of the two two-particle transmission amplitudes $a_{1}^{\dagger} a_{2}^{\dagger} \rightarrow b_{1}^{\dagger} b_{2}^{\dagger}$ and $a_{1}^{\dagger} a_{2}^{\dagger} \rightarrow b_{2}^{\dagger} b_{1}^{\dagger}$ in (12), known as the "Hong-Ou-Mandel (HOM)" (or "Shih-Alley") effect (151, 152), and the second fundamental building block which complements Young's double slit when considering manyparticle transport.

Note that the prediction of (12) differs dramatically from the prediction for distinguishable (classical) particles, where the coincident output event would occur with probability $1 / 2$. Also note that, notwithstanding this completely different probability distribution for two-particle output events, the single mode particle density expectation values $\left\langle b_{o}^{\dagger} b_{o}\right\rangle$ are identical (unity) for classical particles or bosons. This highlights the fact that manyparticle interference effects cannot be witnessed on the level of single-channel observables, but require a correlation measurement (such as the measurement of the coincident event's probability) between distinct output ports (148). ${ }^{10}$ Consequently, we can expect that, for a general unitary $U$ and arbitrary $M \geq N$, quantum transport across the sample represented by $U$ will exhibit dramatic signatures of the particles' indistinguishability, in multi-channel correlation functions measured on output. Mutatis mutandis, as the particles loose their indistinguishability, e.g. by adding an additional degree of freedom which allows for the distinction of sub-groups of particles (in the language of decoherence theory, this is tantamount of providing Welcher Weg information, now in the space of many-particle trajectories), these signatures of many-particle interferences must fade away. This has indeed been demonstrated experimentally, though with the further subtlety that many-particle interference signals do in general exhibit a non-monotonous distinguishability transition, if the latter is controlled by a single continuous parameter (38).

\subsection{Many-particle transport across large, disordered networks}

This brings us back to our overarching theme of disordered networks. When injecting an indistinguishable many particle state of type (9) into a disordered network described by a unitary $U$, see Fig. 5 , we expect strong signatures of many-particle interference on output, according to our above considerations. At a first glance, this is reminiscent of the interference-induced transmission fluctuations which we described in the previous chapter, since, the larger the network, the larger the number of interfering transmission amplitudes. In the present multi-particle case, however, a severe additional problem kicks in: Not only does the number of transmission amplitudes proliferate, but now also the dimension of the space of output states increases exponentially with the particle number. Indeed, the computational effort to evaluate the transition probability between some many-particle input state $\mid$ in $\rangle$ and another many-particle output state $\mid$ out $\rangle$ amounts to evaluating

- the permanent of a real matrix, for distinguishable particles;

- the determinant of a complex matrix, for fermions;

- and the permanent of a complex matrix, for bosons.

While efficient algorithms (in the sense of algorithmic complexity theory) are able to efficiently simulate distinguishable particles and fermions, simulation of the bosonic case is considered to be computationally hard (150).

\footnotetext{
${ }^{10}$ Nowadays, HOM has become a diagnostic tool in photonics, when the indistinguishability of photons needs to be certified. The vanishing of the coincident event probability is a sensitive interference-based - test of the precision of the two-photon state's preparation.
}

HOM:

Hong-Ou-Mandel or Shih-Alley effect 


\section{MANY-PARTICLE INTERFERENCE WITH/OUT DISTINCTIVE DEGREES OF FREEDOM}

Additional degrees of freedom can be added by structuring the single-particle Hilbert space $\mathcal{H}=\mathcal{H}_{\text {modes }} \otimes$ $\mathcal{H}_{\text {internal }}$, where $\mathcal{H}_{\text {modes }}$ represents the mode structure of the main text and $\mathcal{H}_{\text {internal }}$ represents all additional degrees of freedom. In order to correctly populate Fock space, one defines new, non-orthogonal, creation and annihilation operators: $\left[a_{i}(\phi), a_{j}^{\dagger}(\psi)\right]=\langle\phi \mid \psi\rangle \delta_{i j}$, where $|\phi\rangle,|\psi\rangle \in \mathcal{H}_{\text {internal }}$ and $i$ and $j$ label the modes. Since these additional degrees of freedom are assumed to be untouched by the propagation through the network, applying this to the HOM setup, results in

$$
a_{1}^{\dagger}(\phi) \rightarrow\left(b_{1}^{\dagger}(\phi)+b_{2}^{\dagger}(\phi)\right) / \sqrt{2}, a_{2}^{\dagger}(\psi) \rightarrow\left(b_{1}^{\dagger}(\psi)-b_{2}^{\dagger}(\psi)\right) / \sqrt{2} .
$$

When performing a measurement, one typically does not consider the internal degrees of freedom and, therefore, the probability $P_{1,1}$ to measure the particles in different modes is given by

$$
P_{1,1}=\frac{1}{4} \sum_{k, l}\left|\left\langle\Omega\left|b_{1}\left(\eta_{k}\right) b_{2}\left(\eta_{l}\right)\left(b_{1}^{\dagger}(\phi)+b_{2}^{\dagger}(\phi)\right)\left(b_{1}^{\dagger}(\psi)-b_{2}^{\dagger}(\psi)\right)\right| \Omega\right\rangle\right|^{2}=\frac{1}{2}\left(1-|\langle\phi \mid \psi\rangle|^{2}\right),
$$

where $\left\{\eta_{i}\right\}$ form a basis of $\mathcal{H}_{\text {internal. }}$ The result for distinguishable particles is recovered whenever $|\langle\phi \mid \psi\rangle|^{2}=$ 0 . On the other hand, when the particles cannot be distinguished by this internal degree of freedom, we obtain the result for pure, indistinguishable bosons.

\section{BOSON SAMPLING}

Complexity is a widely used term, which is attributed somewhat different - community-dependent - flavours. In the natural sciences, complexity is associated with chaos, disorder, pattern formation, and the (computational or experimental) overhead to fully characterise a complex system's state. In computer sciences, it is the complexity of algorithms which is assessed. Though rather distinct fields, the physics of complex systems and computational complexity theory recently met in the Boson Sampling problem: It is shown that sampling from the output probability distribution, as generated via complex transport (scattering) of non-interacting bosons through a random medium, is computationally hard - i.e., a full characterization of the output state requires an experimental overhead which increases exponentially with the system size. Consequently, the efficient simulation of many-boson interference implies a collapse of the polynomial hierarchy to the third level (150), what challenges many well established aspects of algorithmic complexity theory. In turn, under the perspective of many-particle transport theory, BosonSampling sheds light on rather fundamental aspects of the quantum statistical implications for many-particle dynamics.

In other words, the exhaustive quantitative characterization of $\mid$ out $\rangle$ very quickly exhausts any computational device, as well as a dedicated experimentalist, and thus renders a statistical treatment imperative, much as in the case of classical thermodynamics: We need to identify statistically robust signatures of the many-particle transport, which can be read off from experimental observables with a measurement overhead which scales in a benign way with the size of the problem. Note that this also defines a somewhat 


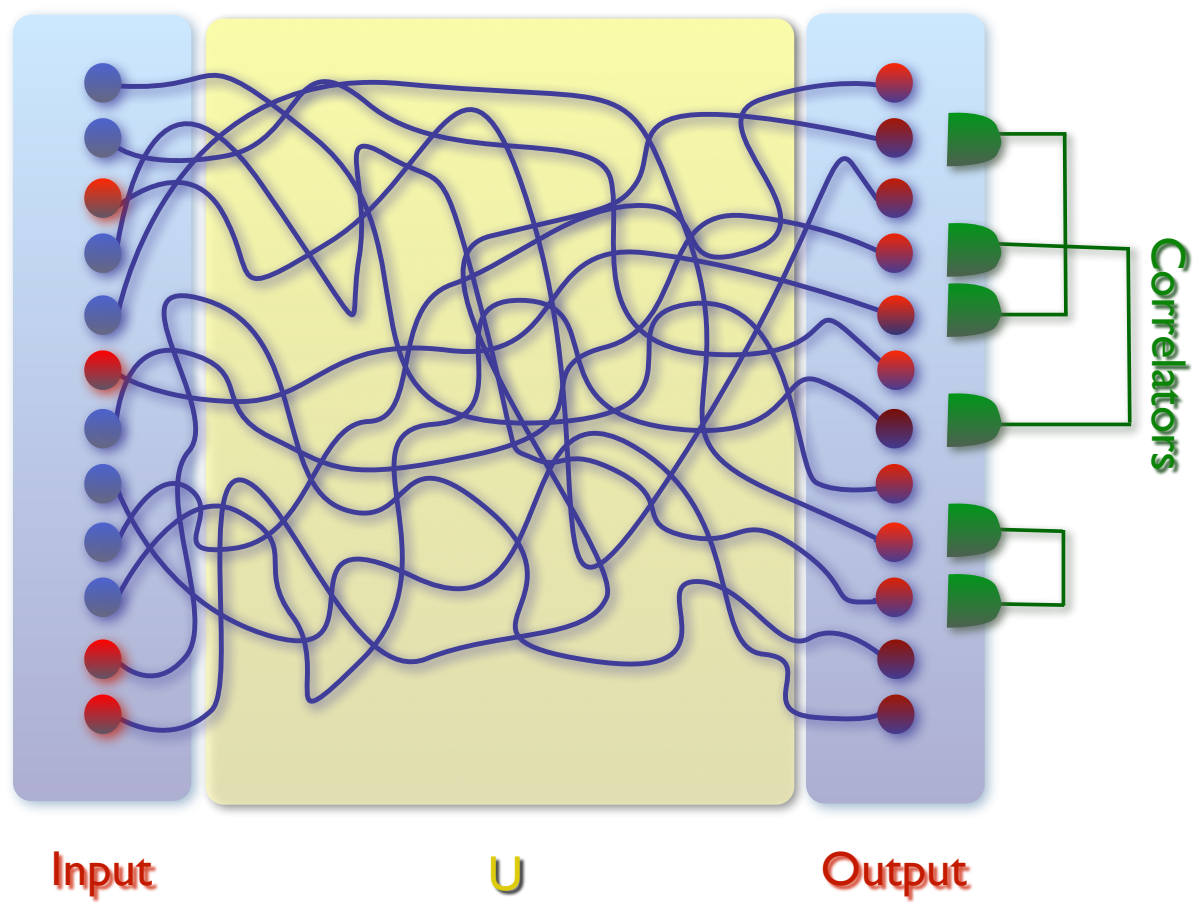

Figure 5

Many-particle transport scenario across a random network represented by a unitary random matrix U sampled over the Haar measure. Particles of variable quantum statistical nature distinguishable, fermionic, bosonic - are prepared in the input state $\mid$ in $\rangle$, defined by (9), and injected at the red input sites, with no more than one particle per site (to allow for a direct comparison of fermionic and bosonic transport properties). Since the computational overhead may grow exponentially with the number of particles and the size of the system, an exhaustive characterization of the many-particle output state is in general prohibitive (see text). For a statistical characterization, two-point correlation functions (18) between all pairs of distinct output sites are sampled over, to build the $C$-data set, which then can be characterized by its lower statistical moments (19).

paradigmatic incident of quantum simulation and of the associated certification problem $(153,154,155,156,157,158,159)$ : A photonic circuit which implements $U$ and prepares |in $\rangle$ will produce output states which cannot be generated computationally, and, in this sense, simulates a problem out of reach for computers. However, for the very same reason, it is virtually impossible to certify that the device constructed by the experimentalist does indeed implement $U$ and operates on $\mid$ in $\rangle$, at least if the only way of certification consisted in the - experimental or computational - verification of the complete output state.

Depending on the unitary $U$ to be implemented, and on the input state $\mid$ in $\rangle$ to be prepared, the certification problem can have different concretisations. If $U$ exhibits some symmetries, these in general (also see our earlier considerations in this review) must manifest themselves in suitably chosen transport quantifiers. Furthermore, to fully characterize the sample, a single input state will in general not suffice, due to the weights $\left\langle\mathcal{E}_{j}\right|$ in $\rangle$ (see (4)), 


\section{HAAR MEASURE}

Probability measures occur in many forms and with many structures, some more natural than others. Perhaps the most natural of all is the uniform distribution that appoints the same weight to all elements in the set under consideration. A type of sets which is often treated in measure theory and has particular relevance in physics are compact topological groups. It can be shown (161) that, for any compact topological group $\mathcal{G}$, there exists a unique measure $\mu$, called the Haar measure, with properties

$$
\begin{aligned}
& \mu(\mathcal{G})=1, \\
& \mu(\mathcal{O})>0 \quad \text { for every nonempty open set } \mathcal{O} \subset \mathcal{G}, \\
& \mu(g \mathcal{M})=\mu(\mathcal{M} g)=\mu(\mathcal{M}) \quad \text { for all } g \in \mathcal{G} \text { and every measurable set } \mathcal{M} \subset \mathcal{G} .
\end{aligned}
$$

Due to the last property, this measure can be interpreted as the uniform distribution on such groups. In quantum transport theory and quantum chaos, one often considers the group of all $N \times N$ unitary matrices, $\mathcal{U}(N)$, on which a Haar measure can be constructed. In Random Matrix Theory, the set of unitary matrices equipped with its Haar measure is also referred to as the Circular Unitary Ensemble (122).

where $\left|\mathcal{E}_{j}\right\rangle$ are the eigenvectors of $\mathcal{U}$, which (implicitly) enter (10). Thereby, only that part of the spectrum of $\mathcal{U}$ is probed which is selected by the local density of states $\mid\left\langle\mathcal{E}_{j}\right|$ in $\rangle\left.\right|^{2}$. $^{11}$ Consequently, one needs to seek certification on the basis of quantities which are obtained by taking averages over the realization of $U$ (and hence of $\mathcal{U}$ ) or/and over $\mid$ in $\rangle$.

In the following, we elaborate on the case of random $U$ taken from the Haar measure, such as to mimic a completely unstructured random network, and input states of type (9).

We seek to certify the specific dynamical signature of the injected particles' quantum statistics by characteristic interference structures in the many-body transmission signal. As we saw above at the elementary example of the HOM set-up, the quantum statistical nature impacts strongly already on the level of two-point correlation functions. While we assume that $M$ and $N$ be significantly larger than two in our present case, we will indeed build a statistical data set from two-point correlation functions evaluated on $\mid$ out $\rangle$. Beyond the intuition gained from HOM, another reason why this can be expected to bear a clear signature of the interfering particles' nature (with the interfering amplitudes defined by the specific choice of $U$ ) is the different number of interfering many-particle amplitudes for different particle species, as expressed by the above-mentioned, distinct mathematical structures of the transition probabilities from $\mid$ in $\rangle$ to $\mid$ out $\rangle$ : The number of interference terms is largest in the bosonic case, and this should, in a sense to be quantified, produce the "most structured" transmission signal. Our statistical quantity to sample is thus chosen

\footnotetext{
${ }^{11}$ Only if a "generic" input state, i.e. a state sufficiently different from an eigenstate of $\mathcal{U}$ and not too close to the edge of the spectrum, has an essentially flat local density of states, under a suitable average, a single input state will suffice. This condition is largely equivalent to requiring that $U$ be generated by a random Hamiltonian chosen from a random matrix ensemble, or that an underlying classical phase space exhibit strictly hyperbolic structure. More recently, this property is being re-discussed under the key word "eigenstate thermalization" (160). 


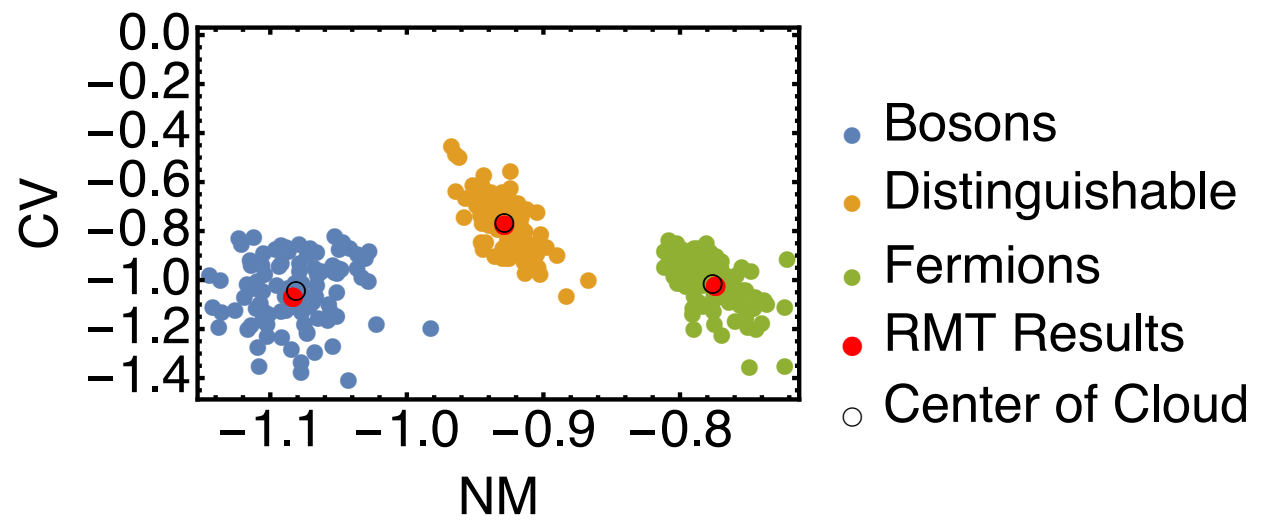

Figure 6

Statistical certification of the transmitted particles' specific scattering dynamics, as determined by the scattering matrix $U$ and the particles' quantum statistical nature. Quantifiers are the normalised mean, NM, and the coefficient of variation, CV, of the C-data set generated by sampling the correlation function $C_{i, j}$, see (18), over all output ports $i \neq j$ of the network (see Fig. 5), for $N=3$ particles fed into an array of $M=13$ input ports. The coloured scattered data points result from direct numerical simulation, for one single random unitary and variations of the input ports populated by the $N$-particle input state (9). Mean values thereof are indicated by the open circles, which coincide with the analytical RMT prediction (153) (given by rather bulky rational expressions in $N$ and $M$ ). This latter agreement implies yet another confirmation of the Bohigas-Giannoni-Schmidt conjecture.

to be the two-point correlator

$$
C_{i, j}=\left\langle n_{i} n_{j}\right\rangle-\left\langle n_{i}\right\rangle\left\langle n_{j}\right\rangle, i \neq j \in[1 ; M], n_{i}=b_{i}^{\dagger} b_{i},
$$

to provide us with the $C$-data set. Given $M, N$, and a realization of $U,(18)$ as well as its lower statistical moments can be evaluated analytically, to finally obtain closed expressions (slightly bulky, but trivially evaluable rational functions of $N$ and $M$ ) for the normalized mean $\mathrm{NM}$, the coefficient of variation $\mathrm{CV}$, and the skewness $\mathrm{S}$ of the $\mathrm{C}$-data set, defined as

$$
\begin{aligned}
& \mathrm{NM}=\frac{\mathbb{E}_{U}(C) M^{2}}{N} \\
& \mathrm{CV}=\frac{\sqrt{\mathbb{E}_{U}\left(C^{2}\right)-\mathbb{E}_{U}(C)^{2}}}{\mathbb{E}_{U}(C)}, \\
& \mathrm{S}=\frac{\mathbb{E}_{U}\left(C^{3}\right)-3 \mathbb{E}_{U}(C) \mathbb{E}_{U}\left(C^{2}\right)+2 \mathbb{E}_{U}(C)^{3}}{\left(\mathbb{E}_{U}\left(C^{2}\right)-\mathbb{E}_{U}(C)^{2}\right)^{3 / 2}},
\end{aligned}
$$

with $\mathbb{E}_{U}$ the average over the unitary group under the Haar measure. Fig. 6 clearly demonstrates, for $N=6, M=13$, respectively, that the different particle species are statistically perfectly well discernable, and that the RMT prediction derived from (19) perfectly fits the numerically generated data.

We thus succeeded to devise a set of statistical, easily computable and measurable quantifiers which unambiguously certify the transmitted particle species - through the characteristic features of many-particle interference signals generated by random unitaries (153). When the underlying dynamics exhibits additional structure or symmetries, we expect certification to be eased (159), and thus have here sketched how to deal with some sort of 
worst case scenario. Blending structural and disorder features will be the next natural step to further refine our understanding of the dynamical consequences of indistinguishability.

\section{SUMMARY POINTS}

1. Quantum transport on finite disordered networks is dominated by strong, interference-induced fluctuations.

2. The statistics of characteristic transport coefficients can be controlled by imposing coarse grained constraints, while maintaining disorder on the microscopic level. By construction, this defines a robust, statistical control strategy for transport on disordered networks.

3. The dynamics of multiple excitations on a network gives rise to characteristic many-body interference effects (to be distinguished from simple bunching or antibunching!), indicative of the quantum statistical nature of the interfering particles, on the level of multi-point correlation functions on output.

4. A statistical analysis of the characteristic interference structures imprinted on loworder correlation functions evaluated on the many-particle output state allows the unambiguous distinction between fermionic, bosonic, and distinguishable particle dynamics, with moderate experimental and computational overhead.

\section{FUTURE ISSUES}

1. Embedding finite disordered networks into superstructures as characteristic, e.g., of the photosynthetic apparatus of algae and plants or also of organic photovoltaic devices, will allow to assess the potential advantage of quantum transport on microscopic scales for the overall functionality.

2. Experimental tools need to be developed which allow to select and possibly knockout specific coherent transition amplitudes,

3. Observables need to be defined which distinguish bunching effects from bona fide bosonic many particle interference contributions.

4. Partial distinguishability $(162,163)$ of the particles can be accounted for by introducing an additional continuous degree of freedom which may convey Welcher Weg information on the level of many-particle transition amplitudes. This will allow to incorporate unavoidable experimental imperfections for boson sampling certification schemes, but also opens novel perspectives for the decoherence theory of many particle quantum systems.

\section{DISCLOSURE STATEMENT}

The authors are not aware of any affiliations, memberships, funding, or financial holdings that might be perceived as affecting the objectivity of this review.

Mattia Walschaers, Frank Schlawin, Thomas Wellens, and Andreas Buchleitner 


\section{ACKNOWLEDGMENTS}

A.B. thanks Uzy Smilansky, Rami Band \& colleagues for one week of discussions on random graphs back in 2009, in Freiburg, what somewhat seeded our interest in this direction. We are grateful to Torsten Scholak, Roberto Mulet, Greg Scholes, Rienk van Grondelle, Tjaart Krueger, Richard Cogdell, Bruno Robert, Tönu Pullerits, Harald Kauffmann, Dominique Delande, Shaul Mukamel, Graham Fleming, Birgitta Whaley for many, and often nicely engaged controversial debates on the possible role of quantum effects for biological functionality. Furthermore, we would like to give credit to Juan-Diego Urbina, Jack Kuipers and Klaus Richter for the fruitful cooperation on Boson Sampling and related issues. M.W. and F.S. acknowledge financial support by the German National Academic Foundation.

\section{References}

1. Imry Y. 2009. Introduction to mesoscopic physics. Oxford: Oxford University Press

2. May V, Kühn O. 2011. Charge and energy transfer dynamics in molecular systems. Weinheim: Wiley-VCH, 3rd ed.

3. Nitzan A. 2006. Chemical dynamics in condensed phases: relaxation, transfer and reactions in condensed molecular systems. Oxford graduate texts. Oxford ; New York: Oxford University Press

4. d'Arcy MB, Godun RM, Summy GS, Guarneri I, Wimberger S, et al. 2004. Phys. Rev. E 69:027201

5. Wimberger S, Guarneri I, Fishman S. 2004. Phys. Rev. Lett. 92:084102

6. Johnson TH, Clark SR, Jaksch D. 2014. EPJ Quantum Technol. 1:1-12

7. Schneider C, Porras D, Schaetz T. 2012. Rep. Prog. Phys. 75:024401

8. R. Feynman R. Leighton MS. 2013. The feynman lctures on physics. vol. III, Quantum Mechanics. California Institute of Technology

9. Englert BG. 2013. Eur. Phys. J. D 67:1-16

10. Albada MPV, Lagendijk A. 1985. Phys. Rev. Lett. 55:2692-2695

11. Anderson PW. 1958. Phys. Rev. 109:1492-1505

12. Casati G, Guarneri I, Shepelyansky D. 1988. IEEE Journal of Quantum Electronics 24:14201444

13. Mishchenko MI. 1993. The Astrophysical Journal 411:351-361

14. Moore FL, Robinson JC, Bharucha C, Williams PE, Raizen MG. 1994. Phys. Rev. Lett. 73:2974-2977

15. Schelle A, Delande D, Buchleitner A. 2009. Phys. Rev. Lett. 102:183001

16. Ringot J, Szriftgiser P, Garreau JC, Delande D. 2000. Phys. Rev. Lett. 85:2741-2744

17. Chabé J, Lemarié G, Grémaud B, Delande D, Szriftgiser P, Garreau JC. 2008. Phys. Rev. Lett. 101:255702

18. Bayfield JE, Casati G, Guarneri I, Sokol DW. 1989. Phys. Rev. Lett. 63:364-367

19. Sirko L, Bauch S, Hlushchuk Y, Koch PM, Blümel R, et al. 2000. Physics Letters A 266:331335

20. Galvez EJ, Sauer BE, Moorman L, Koch PM, Richards D. 1988. Phys. Rev. Lett. 61:2011-2014

21. Jörder F, Zimmermann K, Rodriguez A, Buchleitner A. 2014. Phys. Rev. Lett. 113:063004

22. Krug A, Buchleitner A. 2005. Phys. Rev. A 72:061402

23. Maeda H, Gallagher TF. 2004. Phys. Rev. Lett. 93:193002

24. Abrahams E, Anderson PW, Licciardello DC, Ramakrishnan TV. 1979. Phys. Rev. Lett. 42:673-676

25. Hu H, Strybulevych A, Page JH, Skipetrov SE, van Tiggelen BA. 2008. Nat Phys 4:945-948

26. Sperling T, Bührer W, Aegerter CM, Maret G. 2013. Nat Photon 7:48-52

27. Wegner F. 1979. Z Physik B 35:207-210 
28. Roati G, D'Errico C, Fallani L, Fattori M, Fort C, et al. 2008. Nature 453:895-898

29. Fyodorov YV, Ossipov A, Rodriguez A. 2009. J. Stat. Mech. 2009:L12001

30. Rodriguez A, Vasquez LJ, Slevin K, Römer RA. 2010. Phys. Rev. Lett. 105:046403

31. Vollhardt D, Wölfle P. 1980. Phys. Rev. B 22:4666-4679

32. Billy J, Josse V, Zuo Z, Bernard A, Hambrecht B, et al. 2008. Nature 453:891-894

33. Wolf PE, Maret G. 1985. Phys. Rev. Lett. 55:2696-2699

34. Bergmann G. 1984. Physics Reports 107:1-58

35. Kuga Y, Ishimaru A. 1984. J. Opt. Soc. Am. A 1:831-835

36. Peruzzo A, Lobino M, Matthews JCF, Matsuda N, Politi A, et al. 2010. Science 329:1500-1503

37. Tichy MC, Lim HT, Ra YS, Mintert F, Kim YH, Buchleitner A. 2011. Phys. Rev. A 83:062111

38. Ra YS, Tichy MC, Lim HT, Kwon O, Mintert F, et al. 2013. PNAS 110:1227-1231

39. Meinert F, Mark M, Kirilov E, Lauber K, Weinmann P, et al. 2014. Phys. Rev. Lett. 112:193003

40. Murmann S, Bergschneider A, Klinkhamer V, Zürn G, Lompe T, Jochim S. 2015. Phys. Rev. Lett. 114:080402

41. Labeyrie G, de Tomasi F, Bernard JC, Müller C, Miniatura C, Kaiser R. 1999. Phys. Rev. Lett. 83:5266

42. Modugno G. 2010. Rep. Prog. Phys. 73:102401

43. Tichy MC, Tiersch M, de Melo F, Mintert F, Buchleitner A. 2010. Phys. Rev. Lett. 104:220405

44. Shapiro B. 2012. J. Phys. A: Math. Theor. 45:143001

45. Mosk A, Lagendijk A, Lerosey G, Fink M. 2012. Nature Photonics 6:283

46. Ahlbrecht A, Alberti A, Meschede D, Scholz V, Werner A, Werner R. 2012. New J. Phys. 14:073050

47. Kolovsky AR, Buchleitner A. 2003. Phys. Rev. E 68:056213

48. Ponomarev AV, Madroñero J, Kolovsky AR, Buchleitner A. 2006. Phys. Rev. Lett. 96:050404

49. Geiger T, Wellens T, Buchleitner A. 2012. Phys. Rev. Lett. 109:030601

50. Spethmann N, Kindermann F, John S, Weber C, Meschede D, Widera A. 2012. Phys. Rev. Lett. 109:235301

51. Nelson J. 2003. The physics of solar cells. London : River Edge, NJ: Imperial College Press ; Distributed by World Scientific Pub. Co

52. M.-J. Giannoni A. Voros JZJ, ed. 1991. Chaos and quantum physics. Les Houches Summer School of Theoretical Physics. North-Holland

53. Arndt M, Hornberger K. 2014. Nat Phys 10:271-277

54. Kottos T, Smilansky U. 1999. Annals of Physics 274:76-124

55. Smilansky U. 2007. J. Phys. A: Math. Theor. 40:F621

56. Mülken O, Blumen A. 2011. Physics Reports 502:37-87

57. Venegas-Andraca SE. 2012. Quantum Inf Process 11:1015-1106

58. Scholak T, de Melo F, Wellens T, Mintert F, Buchleitner A. 2011. Phys. Rev. E 83:021912

59. Hul O, Ławniczak M, Bauch S, Sawicki A, Kuś M, Sirko S. 2012. Phys. Rev. Lett. 109:040402

60. Mostarda S, Levi F, Prada-Gracia D, Mintert F, Rao F. 2013. Nat Commun 4

61. Walschaers M, Mulet R, Wellens T, Buchleitner A. 2015. Phys. Rev. E 91:042137

62. Barra F, Gaspard P. 2001. Phys. Rev. E 65:016205

63. Fyodorov YV, Savin DV. 2012. Phys. Rev. Lett. 108:184101

64. Gaspard P. 2014. Scholarpedia 9:9806

65. Breuer HP, Petruccione F. 2002. The theory of open quantum systems. Oxford ; New York: Oxford University Press

66. Tichy MC. 2014. J. Phys. B: At. Mol. Opt. Phys. 47:103001

67. Mandel O, Greiner M, Widera A, Rom T, Hänsch TW, Bloch I. 2003. Phys. Rev. Lett. 91:010407

68. Jaksch D, Zoller P. 2005. Annals of Physics 315:52-79

69. Scholak T, Mintert F, Wellens T, Buchleitner A. 2010. Semicond. Semimet. 83:1-38

70. Rodriguez A, Chakrabarti A, Römer RA. 2012. Phys. Rev. B 86:085119 
71. Plenio MB, Huelga SF. 2008. New J. Phys. 10:113019

72. Mohseni M, Rebentrost P, Lloyd S, Aspuru-Guzik A. 2008. The Journal of Chemical Physics 129:174106

73. Ishizaki A, Fleming GR. 2009. PNAS 106:17255-17260

74. Johnson MW, Amin MHS, Gildert S, Lanting T, Hamze F, et al. 2011. Nature 473:194-198

75. Crespi A, Osellame R, Ramponi R, Brod DJ, Galvão EF, et al. 2013. Nat Photon 7:545-549

76. Metcalf BJ, Thomas-Peter N, Spring JB, Kundys D, Broome MA, et al. 2013. Nat Commun 4:1356

77. Günter G, Schempp H, Robert-de Saint-Vincent M, Gavryusev V, Helmrich S, et al. 2013. Science 342:954-956

78. Scholak T, Wellens T, Buchleitner A. 2014. Phys. Rev. A 90:063415

79. Scholes GD, Mirkovic T, Turner DB, Fassioli F, Buchleitner A. 2012. Energy Environ. Sci. 5:9374-9393

80. Ishizaki A, Fleming GR. 2012. Annual Review of Condensed Matter Physics 3:333-361

81. Falke SM, Rozzi CA, Brida D, Maiuri M, Amato M, et al. 2014. Science 344:1001-1005

82. Tamura H, Burghardt I. 2013. J. Am. Chem. Soc. 135:16364-16367

83. Amerongen Hv, Valkunas L, Grondelle Rv. 2000. Photosynthetic excitons. Singapore: World Scientific

84. Bardeen CJ. 2014. Annual Review of Physical Chemistry 65:127-148

85. Blankenship RE. 2002. Molecular mechanisms of photosynthesis. Oxford ; Malden, MA: Blackwell Science

86. Krüger TPJ, Wientjes E, Croce R, Grondelle Rv. 2011. PNAS 108:13516-13521

87. Mukamel S. 2009. Principles of nonlinear optical spectroscopy. No. 6 in Oxford series in optical and imaging sciences. New York: Oxford Univ. Press

88. Winklhofer M, Dylda E, Thalau P, Wiltschko W, Wiltschko R. 2013. Proc. Roy. Soc. B 280:20130853

89. Franco M, Turin L, Mershin A, E.M.C. S. 2011. Proc. Nat. Ac. Sci. U.S.A. 108:3797

90. Christensson N, Kauffmann HF, Pullerits T, Mančal T. 2012. J. Phys. Chem. B 116:7449-7454

91. Hildner R, Brinks D, Nieder JB, Cogdell RJ, Hulst NFv. 2013. Science 340:1448-1451

92. Romero E, Augulis R, Novoderezhkin VI, Ferretti M, Thieme J, et al. 2014. Nat Phys 10:676682

93. Engel GS, Calhoun TR, Read EL, Ahn TK, Mančal T, et al. 2007. Nature 446:782-786

94. Muskens OL, Venn P, van der Beek T, Wellens T. 2012. Phys. Rev. Lett. 108:223906

95. Alicki R, Miklaszewski W. 2012. The Journal of Chemical Physics 136:134103

96. Walschaers M, Fernandez-de-Cossio Diaz J, Mulet R, Buchleitner A. 2013. Phys. Rev. Lett. 111:180601

97. Zech T, Mulet R, Wellens T, Buchleitner A. 2014. New J. Phys. 16:055002

98. Yang M, Fleming GR. 2002. Chemical Physics 275:355-372

99. Nalbach P, Eckel J, Thorwart M. 2010. New J. Phys. 12:065043

100. Olbrich C, Strümpfer J, Schulten K, Kleinekathöfer U. 2011. J. Phys. Chem. B 115:758-764

101. Manzano D. 2013. PLoS ONE 8:e57041

102. Adolphs J, Müh F, Madjet MEA, Renger T. 2007. Photosynth Res 95:197-209

103. Moix J, Wu J, Huo P, Coker D, Cao J. 2011. J. Phys. Chem. Lett. 2:3045-3052

104. de Melo F, Buchleitner A. 2010. In Quantum Information, Computation and Cryptography, eds. F Benatti, M Fannes, R Floreanini, D Petritis, no. 808 in Lecture Notes in Physics. Springer Berlin Heidelberg, 253-276

105. Brumer P, Shapiro M. 2012. PNAS 109:19575-19578

106. Chenu A, Brańczyk A, Scholes G, Sipe J. 2015. Phys. Rev. Lett. 114:213601

107. Sarovar M, Ishizaki A, Fleming GR, Whaley KB. 2010. Nat Phys 6:462-467

108. Lambropoulos P, Nikolopoulos GM, Nielsen TR, Bay S. 2000. Rep. Prog. Phys. 63:455

109. Faisal F. 1987. Theory of multiphoton processes. Berlin, Heidelberg: Springer Berlin Heidel- 
berg

110. Mülken O, Blumen A, Amthor T, Giese C, Reetz-Lamour M, Weidemüller M. 2007. Phys. Rev. Lett. 99:090601

111. Scholak T, Wellens T, Buchleitner A. 2011. J. Phys. B: At. Mol. Opt. Phys. 44:184012

112. T. Scholak T. Wellens AB. 2011. Europhys. Lett. 96:10001

113. Kassal I, Yuen-Zhou J, Rahimi-Keshari S. 2013. J. Phys. Chem. Lett. 4:362-367

114. Jesenko S, Žnidarič M. 2013. The Journal of Chemical Physics 138:174103

115. Wilkins DM, Dattani NS. 2014. arXiv:1411.3654 [physics, physics:quant-ph, q-bio] ArXiv: 1411.3654

116. Kramer B, MacKinnon A. 1993. Rep. Prog. Phys. 56:1469

117. Pichard JL, Zanon N, Imry Y, Douglas Stone A. 1990. Journal de Physique 51:587-609

118. Hansen N. 2006. In Towards a New Evolutionary Computation, eds. JA Lozano, P Larrañaga, I Inza, E Bengoetxea, no. 192 in Studies in Fuzziness and Soft Computing. Springer Berlin Heidelberg, 75-102

119. Pearson BJ, White JL, Weinacht TC, Bucksbaum PH. 2001. Phys. Rev. A 63:063412

120. Khaneja N, Reiss T, Kehlet C, Schulte-Herbrüggen T, Glaser SJ. 2005. Journal of Magnetic Resonance 172:296-305

121. Cantoni A, Butler P. 1976. Linear Algebra and its Applications 13:275-288

122. Akemann G, Baik J, Di Francesco P, eds. 2011. The Oxford handbook of random matrix theory. Oxford ; New York: Oxford University Press

123. Tomsovic S, Ullmo D. 1994. Phys. Rev. E 50:145-162

124. Stöckmann HJ. 2006. Quantum chaos: an introduction. Cambridge: Cambridge Univ. Press, paperback version, (with corr.) ed.

125. Bohigas O, Giannoni MJ, Schmit C. 1984. Phys. Rev. Lett. 52:1-4

126. Buchleitner A, Delande D, Zakrzewski J, Mantegna RN, Arndt M, Walther H. 1995. Phys. Rev. Lett. 75:3818-3821

127. Cogdell R. 2015. Private communication

128. Blümel R, Buchleitner A, Graham R, Sirko L, Smilansky U, Walther H. 1991. Phys. Rev. A 44:4521

129. Hell SW, Wichmann J. 1994. Opt. Lett. 19:780-782

130. Jalabert RA, Baranger HU, Stone AD. 1990. Phys. Rev. Lett. 65:2442-2445

131. Ericson T. 1960. Phys. Rev. Lett. 5:430-431

132. Mančal T, Valkunas L. 2010. New J. Phys. 12:065044

133. Abramavicius D, Voronine DV, Mukamel S. 2008. PNAS 105:8525-8530

134. Beenakker CWJ. 1991. Phys. Rev. B 44:1646-1656

135. Urban E, Johnson TA, Henage T, Isenhower L, Yavuz DD, et al. 2009. Nat Phys 5:110-114

136. Bardeen J, Cooper LN, Schrieffer JR. 1957. Phys. Rev. 108:1175-1204

137. Gross EP. 1961. Nuovo Cim 20:454-477

138. Dalfovo F, Giorgini S, Pitaevskii LP, Stringari S. 1999. Rev. Mod. Phys. 71:463-512

139. Verbeure A. 2011. Many-body boson systems: half a century later. Theoretical and mathematical physics. London ; New York: Springer

140. Will S, Best T, Braun S, Schneider U, Bloch I. 2011. Phys. Rev. Lett. 106:115305

141. Hänsel W, Hommelhoff P, Hänsch TW, Reichel J. 2001. Nature 413:498-501

142. Genske M, Alt W, Steffen A, Werner AH, Werner RF, et al. 2013. Phys. Rev. Lett. 110:190601

143. Kielpinski D, Monroe C, Wineland DJ. 2002. Nature 417:709-711

144. Petta JR, Johnson AC, Taylor JM, Laird EA, Yacoby A, et al. 2005. Science 309:2180-2184

145. Sansoni L, Sciarrino F, Vallone G, Mataloni P, Crespi A, et al. 2012. Phys. Rev. Lett. 108:010502

146. Wenz AN, Zürn G, Murmann S, Brouzos I, Lompe T, Jochim S. 2013. Science 342:457-460

147. Lim YL, Beige A. 2005. New J. Phys. 7:155

148. Mayer K, Tichy MC, Mintert F, Konrad T, Buchleitner A. 2011. Phys. Rev. A 83:062307 
149. Tichy MC, Tiersch M, Mintert F, Buchleitner A. 2012. New J. Phys. 14:093015

150. Aaronson S, Arkhipov A. 2013a. Theory of Computing 9:143-252

151. Shih YH, Alley CO. 1988. Phys. Rev. Lett. 61:2921-2924

152. Hong CK, Ou ZY, Mandel L. 1987. Phys. Rev. Lett. 59:2044-2046

153. Walschaers M, Kuipers J, Urbina JD, Mayer K, Tichy MC, et al. 2014. arXiv:1410.8547 [cond-mat, physics:physics, physics:quant-ph] ArXiv: 1410.8547

154. Bentivegna M, Spagnolo N, Vitelli C, Brod DJ, Crespi A, et al. 2014. Int. J. Quantum Inform. $12: 1560028$

155. Gogolin C, Kliesch M, Aolita L, Eisert J. 2013. arXiv:1306.3995 [quant-ph] ArXiv: 1306.3995

156. Aaronson S, Arkhipov A. 2013b. arXiv:1309.7460 [quant-ph] ArXiv: 1309.7460

157. Carolan J, Meinecke JDA, Shadbolt PJ, Russell NJ, Ismail N, et al. 2014. Nat Photon 8:621626

158. Aolita L, Gogolin C, Kliesch M, Eisert J. 2014. arXiv:140\%.4817 [quant-ph] ArXiv: 1407.4817

159. Tichy MC, Mayer K, Buchleitner A, Mølmer K. 2014. Phys. Rev. Lett. 113:020502

160. Nandkishore R, Huse DA. 2015. Annual Review of Condensed Matter Physics 6:15-38

161. Conway JB. 1997. A course in functional analysis. No. 96 in Graduate texts in mathematics. New York: Springer, 2nd ed.

162. Shchesnovich VS. 2015. Phys. Rev. A 91:013844

163. Tichy MC. 2015. Phys. Rev. A 91:022316 